\title{
THE TOOMRE SEQUENCE REVISITED WITH HST NICMOS: NUCLEAR BRIGHTNESS PROFILES AND COLORS OF INTERACTING AND MERGING GALAXIES ${ }^{1}$
}

\author{
Jörn Rossa, ${ }^{2,3}$ Seppo Laine, ${ }^{4}$ Roeland P. van der Marel, ${ }^{2}$ J. Christopher Mihos, ${ }^{5}$ \\ John E. Hibbard, ${ }^{6}$ Torsten BöKer, ${ }^{7}$ and Ann I. Zabludoff ${ }^{8}$ \\ Received 2006 July 14; accepted 2007 August 20
}

\begin{abstract}
We discuss the near-infrared (NIR) properties of the nuclei in the 11 merging galaxies of the Toomre sequence, based on high spatial resolution $J, H$, and $K$ imaging data using NICMOS on board the Hubble Space Telescope (HST). The observations are less affected by dust extinction than our previous $H S T$ WFPC2 observations and offer higher spatial resolution than existing ground-based NIR data. Nuclear positions are generally found to be consistent with those reported from data in other wave bands. In NGC 7764A we detect for the first time two nuclei with a separation of about $260 \mathrm{pc}$, consistent with its placement in the middle of the merging sequence. We see a marginal trend for the nuclei to become bluer with advancing merger stage, which we attribute to a dispersal of dust at late times in the merging process. Our data also indicate a statistically significant trend for the nuclei in the sequence to become more luminous, within an aperture of fixed physical size and after correcting for dust extinction, with advancing merger stage. We derive $K$-band surface brightness profiles for those nuclei for which the morphology allows a meaningful isophotal analysis, and fit the profiles with a "Nuker" law for comparison with other samples of galaxies observed with HST. The majority of the nuclei have steep profiles that can be characterized as power-law type. In general, the Toomre-sequence galaxies tend to have steeper profiles and higher central luminosity surface densities than E/S0 galaxies. Our findings can be explained if the Toomre-sequence galaxies have newly formed stars that are concentrated toward their centers. We derive $V-K$ color profiles for the nuclei to further address this possibility, but find that the large amounts of dust extinction complicate their interpretation. Overall, our results are consistent with the generic predictions of $N$-body simulations of spiral galaxy mergers. If left to evolve and fade for several gigayears, it is possible that the properties of the Toomre-sequence nuclei would resemble the properties of the nuclei of normal E/S0 galaxies. Our results therefore support the view that mergers of spiral galaxies can lead to the formation of early-type galaxies.
\end{abstract}

Key words: galaxies: evolution — galaxies: formation — galaxies: interactions — galaxies: nuclei — galaxies: spiral

\section{INTRODUCTION}

It is commonly assumed that the merging of two spiral galaxies following a close interaction will eventually result in their transformation into an elliptical galaxy. This outcome was first suggested by the early simulations of Toomre \& Toomre (1972) and later confirmed by various other investigators (e.g., Barnes 1988; Borne \& Richstone 1991). Evidence gathered over the last few decades conclusively shows that at least a fraction of elliptical galaxies were indeed formed by mergers (see, e.g., the review by Schweizer 1998). Such evidence includes the finding from ground-

\footnotetext{
${ }^{1}$ Based on observations made with the NASA/ESA Hubble Space Telescope, obtained at the Space Telescope Science Institute, which is operated by the Association of Universities for Research in Astronomy (AURA), Inc., under NASA contract NAS5-26555. These observations are associated with proposal 9402.

2 Space Telescope Science Institute, 3700 San Martin Drive, Baltimore, MD 21218,USA; marel@stsci.edu.

3 Present address: Department of Astronomy, University of Florida, 211 Bryant Space Science Center, P.O. Box 112055, Gainesville, FL 32611-2055, USA; jrossa@ astro.ufl.edu.

4 Spitzer Science Center, California Institute of Technology, Mail Code 220-6, Pasadena, CA 91125, USA; seppo@ipac.caltech.edu.

${ }^{5}$ Department of Astronomy, Case Western Reserve University, 10900 Euclid Avenue, Cleveland, OH 44106, USA; mihos@ case.edu.

6 National Radio Astronomy Observatory, 520 Edgemont Road, Charlottesville, VA 22903-2475, USA; jhibbard@nrao.edu.

7 Astrophysics Division, RSSD, European Space Research and Technology Centre, NL-2200 AG Noordwijk, The Netherlands; tboeker@rssd.esa.int.

${ }^{8}$ Steward Observatory, University of Arizona, 933 North Cherry Avenue, Tucson, AZ 85721-0065, USA; azabludoff@as.arizona.edu.
}

based imaging that on global scales many merger remnants are well fit by the $R^{1 / 4}$ luminosity profiles that are characteristic of elliptical galaxies (e.g., Schweizer 1982; Wright et al. 1990; Hibbard \& Yun 1999; Rothberg \& Joseph 2004).

Previous studies of spiral-spiral mergers have focused extensively on the tidal tails (e.g., Hibbard \& van Gorkom 1996; Hibbard et al. 2001), globular clusters (e.g., Whitmore et al. 1993; Whitmore \& Schweizer 1995; Schweizer et al. 1996, 2004), young star clusters (e.g., Whitmore et al. 1999, 2005), and dwarf galaxies that may have formed during the merger (e.g., Braine et al. 2001; Weilbacher et al. 2003). Less attention has been paid to the nuclei, however (although see Schweizer 1996; Scoville et al. 2000). This is in contrast with the case for, e.g., the nuclear regions of spiral galaxies (Carollo et al. 1997; Böker et al. 2002) and ellipticals (e.g., Lauer et al. 1995; Ravindranath et al. 2001, hereafter R01; Rest et al. 2001; Laine et al. 2003a; Ferrarese et al. 2006), which have been studied at high resolution using the Hubble Space Telescope (HST). In fact, one of the most important physical processes that occurs during the merging process is the inflow of gas into the nuclear region (e.g., Noguchi 1988; Mihos \& Hernquist 1994, 1996; Barnes \& Hernquist 1996). To study the question of whether and how mergers convert the structure of the nuclear regions from disk galaxy bulges or pseudobulges into the density profiles seen in the centers of elliptical galaxies, it is therefore important to investigate the nuclear regions at the highest achievable angular resolution.

Toomre (1977) assembled a small sample of prospective merger systems in various stages of merging that later became known as 
TABLE 1

The Nuclei of the Toomre Sequence

\begin{tabular}{|c|c|c|c|c|c|c|c|c|c|}
\hline $\begin{array}{l}\text { Seq. No. } \\
\text { (1) }\end{array}$ & $\begin{array}{l}\text { Galaxy } \\
\text { (2) }\end{array}$ & $\begin{array}{l}\text { Alt. Name } \\
\text { (3) }\end{array}$ & $\begin{array}{l}\text { R.A. (J2000.0) } \\
\text { (4) }\end{array}$ & $\begin{array}{c}\text { Decl. (J2000.0) } \\
\text { (5) }\end{array}$ & $\begin{array}{l}\text { Comments on } \\
\text { Nuc. Morph. } \\
\text { (6) }\end{array}$ & $\begin{array}{c}c z \\
\left(\mathrm{~km} \mathrm{~s}^{-1}\right) \\
(7)\end{array}$ & $\begin{array}{c}\text { Distance } \\
(\mathrm{Mpc}) \\
(8)\end{array}$ & $\begin{array}{l}0.1^{\prime \prime} \\
(\mathrm{pc}) \\
(9)\end{array}$ & $\begin{array}{l}\text { Class } \\
(10)\end{array}$ \\
\hline \multicolumn{10}{|c|}{ Early Stage } \\
\hline $1 \ldots \ldots \ldots$ & NGC 4038 & Arp 244 & 120153.06 & -185203.6 & $\mathrm{c}, \mathrm{d}, \mathrm{f}$ & 1616 & 13.8 & 7 & $\ldots$ \\
\hline 1 & NGC 4039 & Arp 244 & 120153.61 & $\begin{array}{lll}-18 & 53 & 10.7\end{array}$ & $\mathrm{p}$ & 1624 & 13.8 & 7 & $\ldots$ \\
\hline $2 \ldots \ldots \ldots \ldots \ldots \ldots$ & NGC 4676A & Arp 242 & 124610.09 & +304354.6 & $\mathrm{p}+\mathrm{s}, \mathrm{b} ?$ & 6613 & 88.2 & 43 & LINER \\
\hline $2 \ldots \ldots \ldots \ldots \ldots \ldots$ & NGC 4676B & Arp 242 & 124611.21 & +304322.2 & $\mathrm{p}$ & 6613 & 88.2 & 43 & LINER \\
\hline З & NGC 7592 W & VV 731 & 231821.78 & -042458.0 & $\mathrm{p}+\mathrm{r}$ & 7280 & 97.1 & 47 & Sy2 \\
\hline 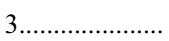 & NGC 7592 E & VV 731 & $\ldots$ & $\ldots$ & $c, e, f$ & 7280 & 97.1 & 47 & $\ldots$ \\
\hline 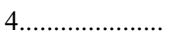 & NGC 7764A E & AM $2350-410$ & 235323.75 & -404825.2 & $p+f$ & 9162 & 122.2 & 59 & $\ldots$ \\
\hline $4 \ldots \ldots \ldots \ldots$ & NGC 7764A W & AM $2350-410$ & 235323.72 & -404825.4 & $\mathrm{p}$ & 9162 & 122.2 & 59 & $\ldots$ \\
\hline \multicolumn{10}{|c|}{ Midstage } \\
\hline $5 \ldots \ldots$ & NGC 6621 & Arp 81 & 181255.34 & +682148.8 & $\mathrm{p}+\mathrm{c}, \mathrm{e}$ & 6191 & 84.4 & 41 & $\ldots$ \\
\hline $5 \ldots \ldots \ldots \ldots \ldots \ldots$ & NGC 6622 & Arp 81 & 181259.40 & +682115.0 & $\mathrm{p}$ & 6466 & 84.4 & 41 & $\ldots$ \\
\hline 6.................... & NGC 3509 & Arp 335 & 110423.63 & +044943.9 & $\mathrm{p}$ & 7704 & 102.7 & 50 & $\ldots$ \\
\hline 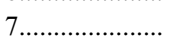 & NGC $520 \mathrm{~S}$ & Arp 157 & 012434.91 & +034729.1 & $\mathrm{p}$ & 2281 & 30.4 & 15 & $\ldots$ \\
\hline 7 ......................... & NGC $520 \mathrm{~N}$ & Arp 157 & 012433.29 & +034802.6 & $\mathrm{p} ?, \mathrm{c}$ & 2281 & 30.4 & 15 & $\ldots$ \\
\hline \multicolumn{10}{|c|}{ Late Stage } \\
\hline $8 \ldots \ldots \ldots \ldots \ldots \ldots$ & NGC 2623 & Arp 243 & 083824.14 & +254516.1 & $\mathrm{p}$ & 5535 & 73.8 & 36 & LINER \\
\hline 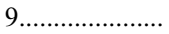 & NGC $3256 \mathrm{~N}$ & VV 65 & 102751.17 & -435415.6 & $\mathrm{p}$ & 2738 & 36.5 & 18 & $\ldots$ \\
\hline 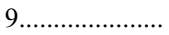 & NGC $3256 \mathrm{~S}$ & VV 65 & 102751.16 & -435420.8 & $\mathrm{e}$ & 2738 & 36.5 & 18 & $\ldots$ \\
\hline $10 \ldots \ldots \ldots \ldots \ldots \ldots$ & NGC 3921 & Arp 224 & 115107.19 & +550443.1 & $\mathrm{p}$ & 5838 & 77.8 & 38 & LINER \\
\hline $11 \ldots \ldots \ldots \ldots \ldots \ldots$ & NGC 7252 & Arp 226 & 222044.77 & -244042.6 & $\mathrm{p}, \mathrm{s}$ & 4688 & 62.5 & 30 & $\ldots$ \\
\hline
\end{tabular}

Notes.-Col. (1): Toomre-sequence number. Cols. (2) and (3): Galaxy name and alternate name. Cols. (4) and (5): Coordinates of the nuclei as measured on our NICMOS F205W and F222M images. Units of right ascension are hours, minutes, and seconds, and units of declination are degrees, arcminutes, and arcseconds. The $1 \sigma$ absolute accuracy of these coordinates is $\sim 1.6^{\prime \prime}$. Col. (6): Nuclear morphology (as seen in the FOV of the NIC2 images, which typically extend to $\sim 10^{\prime \prime}$ from the galaxy nucleus), with the following nomenclature: (b) bar; (c) complex; (d) dusty; (e) extended; (f) filamentary; (p) pointlike (compact); (r) ring; (s) nuclear spiral. Col. (7): Heliocentric velocity, taken from the NASA/IPAC Extragalactic Database, except for NGC 4676, whose value was taken from the Lyon Extragalactic Database. Col. (8): Distance, calculated using $H_{0}=75 \mathrm{~km} \mathrm{~s}^{-1} \mathrm{Mpc}^{-1}$. For the NGC 6621/6622 system we have calculated the mean distance by averaging both distances calculated from the velocities. For NGC 4038/4039 we adopted the distance derived from a measurement of the tip of the red giant branch (Saviane et al. 2004). Col. (9): Physical scale in parsecs corresponding to $0.1^{\prime \prime}$. Col. (10): Spectral classification for all galaxies with possible AGN contributions. These classifications were compiled from various literature sources, as described in Paper I. We note that this classification is by no means complete, since not all galaxies in the Toomre sequence have been studied in detail (or at all) spectroscopically. The position of the true nucleus of NGC $7592 \mathrm{E}$ is difficult to assess from our NIR images, as it has an extended morphology with two maxima, and other parts of the circumnuclear region are not covered by our aperture.

the Toomre sequence. We investigated various properties of this optically selected merger sample with $H S T$ WFPC2, in particular the response of the nuclei to the merging process (Laine et al. 2003b, hereafter Paper I). However, in the optical several of the nuclear regions are hidden behind dust, which makes identification of the nuclei difficult, if not impossible. Near-infrared (NIR) observations are much less affected by dust, and thus are better suited to identifying the location of the nuclei within the often complex circumnuclear environments of the host galaxies.

Previous investigations have studied a range of basic properties of various merger samples (e.g., Joseph et al. 1984; Lonsdale et al. 1984; Stanford \& Bushouse 1991; Bushouse \& Stanford 1992; Rothberg \& Joseph 2004). Most of the Toomre-sequence galaxies have been studied previously from the ground at NIR wavelengths: NGC 4038/4039 (Bushouse \& Werner 1990; Hibbard et al. 2001; Kassin et al. 2003), NGC 4676 (J. Hibbard 2006, unpublished), NGC 7592 (Bushouse \& Werner 1990), NGC 6621/6622 (Bushouse \& Werner 1990), NGC 3509 (Bushouse \& Stanford 1992), NGC 520 (Bushouse \& Stanford 1992; Kotilainen et al. 2001), NGC 3256 (Kotilainen et al. 1996), and more recently, all four late-stage mergers (Rothberg \& Joseph 2004). However, these observations either lacked adequate spatial resolution to reveal the detailed structure of the nuclei, or were aimed at investigating prop- erties on larger scales. In order to explore the nuclear regions themselves, the high spatial resolution of the $H S T(5-50 \mathrm{pc}$ at the distance of the Toomre-sequence galaxies) is imperative.

We present here a study of the entire Toomre sequence with HST NICMOS. This work is part of a high spatial resolution multiinstrument $H S T$ survey of the nuclear regions in the Toomre sequence (cf. Table 1), including WFPC2, NICMOS, and STIS observations. Earlier (see Paper I) we reported on the nuclear morphologies and luminosity densities based on WFPC2 broad- and narrowband observations. In a future paper (J. Rossa et al. 2008, in preparation, hereafter Paper III) we will present stellar population studies based on STIS G430L spectra. In this paper we focus on the nuclear morphology and photometric properties as revealed by the NICMOS observations. We make a detailed comparison with our previous WFPC2 observations and other existing multiwavelength observations, and discuss the results in the context of the evolution of merging galaxies and the formation of ellipticals.

Our investigation is the first comprehensive high spatial resolution study of an optically selected sample of mergers. Merging galaxies often have high infrared luminosities (Sanders et al. 1988), sometimes in excess of $L_{\mathrm{IR}} \geq 10^{11} L_{\odot}$, and samples of LIRGS (luminous infrared galaxies) and ULIRGS (ultraluminous infrared galaxies) are dominated by merging systems (Sanders \& 
Mirabel 1996). The present optically selected sample thus provides a useful comparison to HST NICMOS studies of merger remnants selected only on the basis of their infrared luminosity (e.g., Scoville et al. 2000; Borne et al. 1999).

This paper is structured in the following way: in $\S 2$ we present the observations and the data reduction procedures. In $\S 3$ we show results on the nuclear morphology and color mapping for each individual system separately (grouped in three merger stages), and compare them with other multiwavelength studies. In $\S 4$ we present and discuss photometry, colors, and radial color profiles for the Toomre nuclei. In $\S 5$ we present $K$-band surface brightness profiles, and discuss the results in the context of the evolution of merging galaxies and the formation of early-type galaxies. In $\S 6$ we summarize the main results of the paper.

\section{SAMPLE AND OBSERVATIONS}

\subsection{Sample}

Our sample is the Toomre sequence of relatively nearby $(D \leq$ $122 \mathrm{Mpc}$ ), strongly interacting and merging galaxies, ordered according to the estimated degree of completeness of the merging process (Toomre 1977). The basic properties of the 11 systems are listed in Table 1. Distances in the table and throughout this paper are based on a Hubble constant of $H_{0}=75 \mathrm{~km} \mathrm{~s}^{-1} \mathrm{Mpc}^{-1}$, save for the distance to the Antennae, which is based on observations of red giant branch stars (Saviane et al. 2004). The systems span a wide range of dynamical phases, from galaxies early in the merging process (e.g., NGC 4038/4039) to late-stage systems (merger remnants; e.g., NGC 7252). A detailed description of the optical morphology of the nuclear regions is presented in Paper I. We emphasize that the Toomre sequence was chosen originally based on the visual inspection of photographic plates, mostly those presented in the Atlas of Peculiar Galaxies (Arp 1966), and the rough merger stage has been derived from the degree of coalescence (Toomre 1977). Although the very early and very late stages can be distinguished quite unambiguously, an exact placement in a sequence may be possible only after deriving the dynamical history of the merger through detailed simulations.

\subsection{Observations and Data Reduction}

All NIR images discussed in this paper were obtained with the Near Infrared Camera and Multi-Object Spectrometer (NICMOS) on board $H S T$, which uses a $256 \times 256$ pixel $\mathrm{HgCdTe}$ array. We used the NIC2 camera, which has a field of view (FOV) of $19.2^{\prime \prime} \times 19.2^{\prime \prime}$ and a pixel scale of $0.075^{\prime \prime} \mathrm{pixel}^{-1}$. We observed seven of the 11 Toomre systems in three filter passbands (F110W, F160W, and F205W), which resemble but do not quite match the ground-based $J, H$, and $K$ bands. In addition, we made use of archival NICMOS images of the remaining four late-stage Toomre galaxies (NGC 2623, NGC 3256, NGC 3921, and NGC 7252), which originated from the following GO programs: NGC 2623: program 7219 (PI: N. Scoville); NGC 3256: program 7251 (PI: J. Black); and NGC 3921 and NGC 7252: program 7268 (PI: R. van der Marel). All NICMOS observations were performed in the MULTIACCUM observing mode. We note that for NGC 2623 and NGC 3256 no F205W observations have been carried out, so we used available F222M observations instead. Furthermore, for NGC 3256 no F110W observations were available. A complete listing of all the observations is given in Table 2.

Our observations were obtained shortly after NICMOS was reactivated through the installation of a cryocooler (installed on 2002 March 7). After this reactivation, the NICMOS aperture locations shifted slightly with respect to their prior locations in the focal plane. However, the aperture location table used by the tele- scope pointing system was not updated until later in the year. As a result, some of our observations showed an offset of a few arcseconds between the target and the intended location on the detector. This generally was not a problem, since in almost all cases the target still fell on the detector. Only our observations for NGC 7592 were somewhat adversely affected, as discussed in $\S 3.2$ below.

The data obtained in the $\mathrm{F} 110 \mathrm{~W}$ and $\mathrm{F} 160 \mathrm{~W}$ passbands were pipeline-reduced, including the basic reduction steps such as dark and flat-field correction using the STSDAS ${ }^{9}$ tasks calnica and $c a l n i c b$. We used a dither pattern for the observations in order to allow for good cosmic-ray removal and correction of instrumental blemishes such as bad pixels and the "grot" (areas of reduced sensitivity due to paint flecks that have fallen on the detector). For the F205W observations a slightly different observing strategy was used in order to subtract the thermal background emission from the telescope. This is only an issue for observations at or longward of the $K$ band. We used a small-scale dither pattern in combination with a chop between the object and background positions. The chop positions were separated by either $60^{\prime \prime}$ or $90^{\prime \prime}$ (depending on the extent of the galaxy) from the galaxy and contained little or no emission from the galaxy. As this combined strategy is currently not supported in the pipeline reduction, we had to manually adjust a few steps, and edit the respective table files in calnica, before we could go on with processing the images with calnicb. For further details concerning the general reduction steps we refer to the NICMOS Data Handbook (Dickinson et al. 2002). Throughout the remainder of the paper, we refer for simplicity to the F110W, F160W, and F205W/F222M filter bands as the $J, H$, and $K$ bands.

For the photometric comparison with our WFPC2 data and in order to create color maps, we had to register and resample the images so that the WFPC2 and NIC2 images are on the same spatial scale. In most cases we used only the PC chip of the WFPC2 (FOV of $35^{\prime \prime} \times 35^{\prime \prime}$, as compared to $19.2^{\prime \prime} \times 19.2^{\prime \prime}$ for NIC2), on which all the nuclei save for the Antennae were centered. We used the STSDAS task reflresamp to perform the necessary steps, aligning all images to the NIC2 images. Rotations, translations, and scale differences were taken into account using a combination of header information about the telescope pointing and orientation, and matching object positions in the field. We measured the positions of at least two reference points (e.g., stars, compact clusters, or H II regions) in both the WFPC2 and NIC2 images and checked the final registering by blinking the images. In the case of NGC $520 \mathrm{~S}$ and NGC 4676A we note that due to the lack of any reference points in the NIR that had visible counterparts in the optical, the final registering between the WFPC2 and NICMOS images may be off by 2-3 pixels. We did not correct for differences in the FWHM of the point-spread function (PSF) between the optical and infrared data sets; this may cause small artifacts in the central $\sim 0.2^{\prime \prime}$ of the color maps presented below. However, none of the main conclusions of our paper are affected by these artifacts.

Magnitudes were obtained from count rates using $m_{\text {filter }}=$ $-2.5 \log (\mathrm{CR})+\mathrm{ZP}_{\text {filter }}$, where $\mathrm{ZP}_{\text {filter }}$ is the magnitude zero point of the individual filter. Because the NICMOS filters do not correspond exactly to the $J, H$, and $K$ bands, there are color terms in the zero points of the photometric transformation equations. These color terms can vary throughout each galaxy, due to stellar population gradients and the generally very inhomogeneous distributions of dust. To calculate and correct for the color terms on a pixel-by-pixel basis is not straightforward. We have therefore taken a simplified approach. First, we adopted an initial guess zero point

\footnotetext{
9 The Space Telescope Science Data Analysis System is a product of the Space Telescope Science Institute, which is operated by AURA, Inc., for NASA.
} 
TABLE 2

NICMOS OBSERVATIONS

\begin{tabular}{|c|c|c|c|c|c|}
\hline $\begin{array}{l}\text { Galaxy } \\
\text { (1) }\end{array}$ & $\begin{array}{c}\text { Data Set Identifier } \\
\text { (HST Archive) } \\
\text { (2) }\end{array}$ & $\begin{array}{l}\text { Date } \\
\text { (3) }\end{array}$ & $\begin{array}{c}\text { Filter } \\
\text { (4) }\end{array}$ & $\begin{array}{c}\lambda_{\text {cen }} \\
(\mu \mathrm{m}) \\
(5)\end{array}$ & $\begin{array}{l}t_{\exp } \\
(\mathrm{s}) \\
(6)\end{array}$ \\
\hline NGC $4038 \ldots$ & N8FR01010 & 2003 Jun 12 & F110W & 1.1264 & 160 \\
\hline 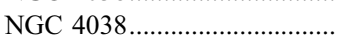 & N8FR01020 & 2003 Jun 12 & F160W & 1.6037 & 160 \\
\hline NGC 4038 & N8FR01030 & 2003 Jun 12 & F205W & 2.0658 & 448 \\
\hline 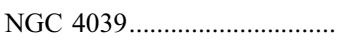 & N8FR02010 & 2003 May 26 & F110W & 1.1264 & 160 \\
\hline 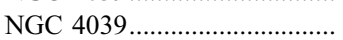 & N8FR02020 & 2003 May 26 & F160W & 1.6037 & 160 \\
\hline 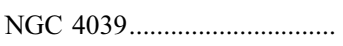 & N8FR02030 & 2003 May 26 & F205W & 2.0658 & 448 \\
\hline 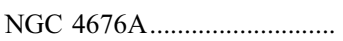 & N8FR03010 & 2003 Jun 12 & F110W & 1.1264 & 192 \\
\hline 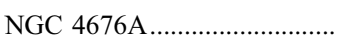 & N8FR03020 & 2003 Jun 12 & F160W & 1.6037 & 160 \\
\hline 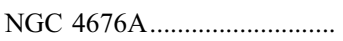 & N8FR03030 & 2003 Jun 12 & F205W & 2.0658 & 448 \\
\hline 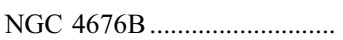 & N8FR04010 & 2003 Jun 21 & F110W & 1.1264 & 192 \\
\hline 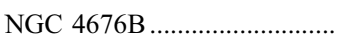 & N8FR04020 & 2003 Jun 21 & F160W & 1.6037 & 160 \\
\hline 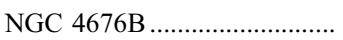 & N8FR04030 & 2003 Jun 21 & F205W & 2.0658 & 448 \\
\hline 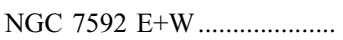 & N8FR05010 & 2002 Oct 4 & F110W & 1.1264 & 160 \\
\hline 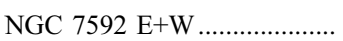 & N8FR05020 & 2002 Oct 4 & F160W & 1.6037 & 160 \\
\hline 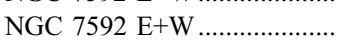 & N8FR05030 & 2002 Oct 4 & F205W & 2.0658 & 448 \\
\hline NGC 7764A E+W ..................... & N8FR06010 & 2003 Apr 24 & F110W & 1.1264 & 192 \\
\hline NGC 7764A E+W ...................... & N8FR06020 & 2003 Apr 24 & F160W & 1.6037 & 192 \\
\hline NGC 7764A E+W & N8FR06030 & 2003 Apr 24 & F205W & 2.0658 & 448 \\
\hline 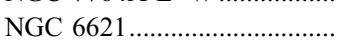 & N8FR07010 & 2002 Aug 30 & F110W & 1.1264 & 224 \\
\hline 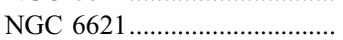 & N8FR07020 & 2002 Aug 30 & F160W & 1.6037 & 224 \\
\hline NGC $6621 \ldots \ldots$ & N8FR07030 & 2002 Aug 30 & $\mathrm{~F} 205 \mathrm{~W}$ & 2.0658 & 448 \\
\hline 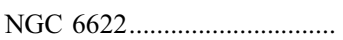 & N8FR08010 & 2002 Aug 28 & F110W & 1.1264 & 224 \\
\hline NGC 6622 & N8FR08020 & 2002 Aug 28 & F160W & 1.6037 & 224 \\
\hline 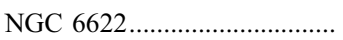 & N8FR08030 & 2002 Aug 28 & $\mathrm{~F} 205 \mathrm{~W}$ & 2.0658 & 448 \\
\hline 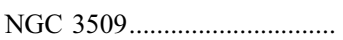 & N8FR09010 & 2002 Oct 30 & F110W & 1.1264 & 160 \\
\hline 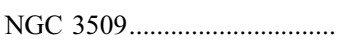 & N8FR09020 & 2002 Oct 30 & F160W & 1.6037 & 160 \\
\hline NGC $3509 \ldots \ldots \ldots \ldots \ldots \ldots \ldots \ldots \ldots \ldots$ & N8FR09030 & 2002 Oct 30 & F205W & 2.0658 & 448 \\
\hline 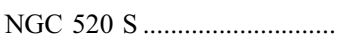 & N8FR10010 & 2003 Jan 18 & F110W & 1.1264 & 160 \\
\hline NGC $520 \mathrm{~S}$ & N8FR10020 & 2003 Jan 18 & F160W & 1.6037 & 160 \\
\hline NGC $520 \mathrm{~S}$ & N8FR10030 & 2003 Jan 18 & F $205 \mathrm{~W}$ & 2.0658 & 448 \\
\hline 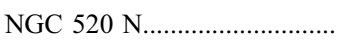 & N8FR11010 & 2003 Jan 18 & F110W & 1.1264 & 160 \\
\hline 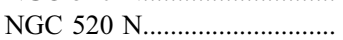 & N8FR11020 & 2003 Jan 18 & F160W & 1.6037 & 160 \\
\hline NGC 520 N & N8FR11030 & 2003 Jan 18 & F205W & 2.0658 & 448 \\
\hline 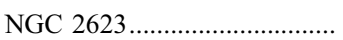 & $\mathrm{N} 48 \mathrm{H} 23010$ & 1997 Nov 19 & F110W & 1.1264 & 88 \\
\hline 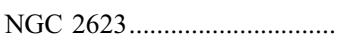 & $\mathrm{N} 48 \mathrm{H} 23020$ & 1997 Nov 19 & F160W & 1.6037 & 88 \\
\hline 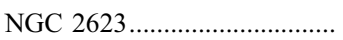 & N48H23030 & 1997 Nov 19 & $\mathrm{~F} 222 \mathrm{M}$ & 2.2177 & 120 \\
\hline 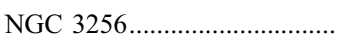 & N4G601080 & 1997 Nov 28 & F160W & 1.6037 & 48 \\
\hline 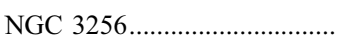 & N4G601090/91 & 1997 Nov 28 & F222M & 2.2177 & 80 \\
\hline 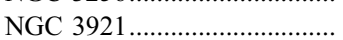 & N49J09040 & 1998 Jan 10 & F110W & 1.1264 & 160 \\
\hline NGC 3921 & N49J09020/60 & 1998 Jan 10 & F160W & 1.6037 & 192 \\
\hline 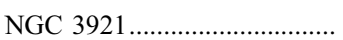 & N49J09080/81/82 & 1998 Jan 10 & F205W & 2.0658 & 256 \\
\hline 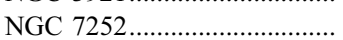 & N49J12040 & 1997 Nov 11 & F110W & 1.1264 & 128 \\
\hline NGC $7252 \ldots \ldots \ldots$ & N49J12020/60 & 1997 Nov 11 & F160W & 1.6037 & 128 \\
\hline 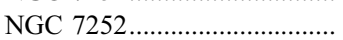 & N49J12080/81/82 & 1997 Nov 11 & F205W & 2.0658 & 256 \\
\hline
\end{tabular}

Notes.-Col. (1): Galaxy identifier. Col. (2): Data set identifier from the HST data archive. Col. (3): Date of observations. Col. (4): HST NICMOS filter used. Col. (5): Central wavelength. Col. (6): Exposure time. Note that for NGC 3256 no F110W observations are available in the archive. The $K$-band observations for NGC 2623 and NGC 3256 were performed with the F222M filter, which covers the CO continuum $(2.15-2.29 \mu \mathrm{m})$.

that depends only on the filter. These zero points were based on the color terms appropriate for a $10^{9} \mathrm{yr}$ old population (as given by Bruzual \& Charlot 2003) without additional reddening. The color terms were calculated using the calcphot task in the STSDAS package SYNPHOT. We then refined the zero points on a galaxyby-galaxy basis. For each galaxy we calculated (as discussed in $\S 4$ ) the count rates in several apertures of fixed sizes. These were combined with the initial-guess zero points to obtain initial guesses for the $J, H$, and $K$ magnitudes. We then used these magnitudes to estimate the colors in the aperture, and subsequently we used these colors to improve the zero-point estimates.
From a variety of tests we infer that this iterative scheme yields magnitudes that are accurate to $\sim 0.05 \mathrm{mag}$ in each of the $J, H$, and $K$ bands. This is generally much smaller than the variations in color either within a given galaxy or between different galaxies. When quoting aperture magnitudes in $\S 4$ we use the zero points calculated specifically for each given combination of filter, galaxy, and aperture. When displaying the images, constructing surface brightness profiles, and constructing color maps, we adopt a single zero point for each combination offilter and galaxy by averaging the zero points calculated for the $100 \mathrm{pc}$ and $1 \mathrm{kpc}$ apertures. Our calibrated magnitudes are in a system defined by the $J_{-}, H-$, and 

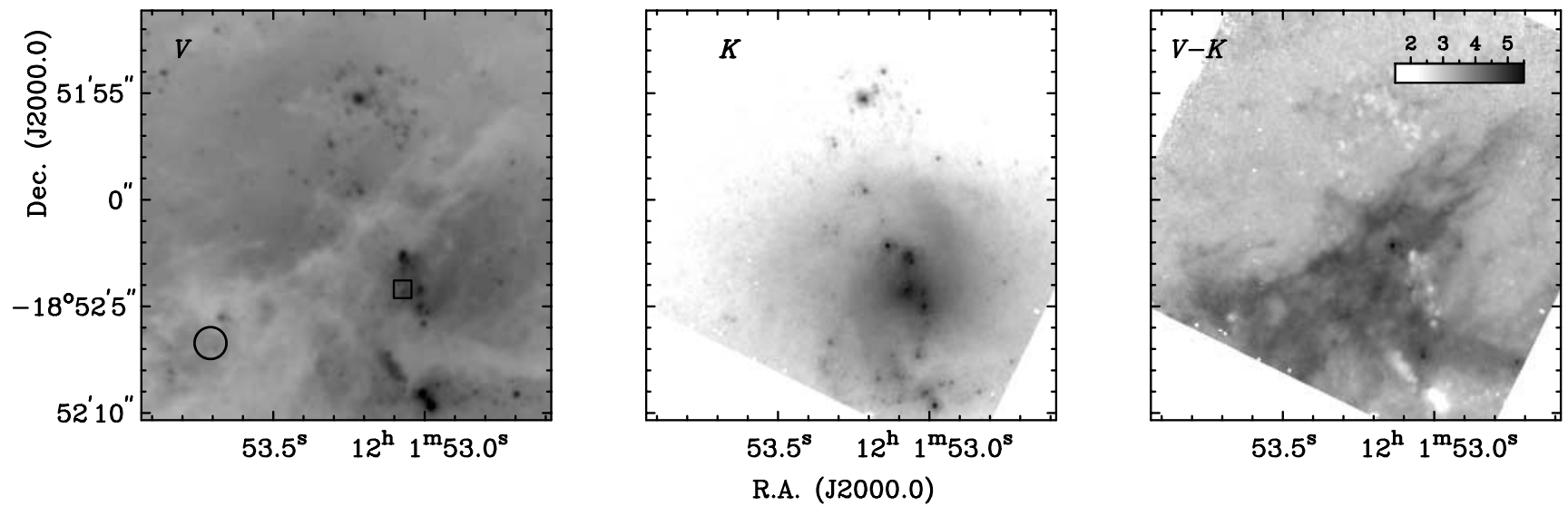

FIg. 1.- $V$-band (left), $K$-band (middle), and $V-K$ (right) images of the circumnuclear region in NGC 4038 . The nucleus is marked by a square in the $V$-band image, as in this particular case several nuclear components are visible. The displayed FOV translates to a physical size of $1.3 \mathrm{kpc} \times 1.3 \mathrm{kpc}$. To visually indicate the scale we show an aperture of diameter $100 \mathrm{pc}$ (solid circle) and, for all nuclei other than the Antennae, also of $1 \mathrm{kpc}$ (dashed circle) at the bottom left of the $V$-band image.

$K$-band filter transmission curves of the Bessell system (Bessell \& Brett 1988).

\section{NUCLEAR MORPHOLOGY}

\subsection{Introduction}

Figures 1 (NGC 4038) through 16 (NGC 7252) show images of the nuclear regions of the Toomre-sequence galaxies. Most figures show three panels arranged as follows: the left panel in each figure shows the WFPC2 F555W ( $V$-band) image from Paper I; the middle panel shows the NICMOS $K$-band image from the present study; and the right panel shows the $V-K$ image. A comparison of the $V$ and $K$ bands provides the longest baseline in color space available from our $H S T$ data. We also created color images of $J-H, J-K$, and $H-K$, composed entirely of the NICMOS data. However, these generally show the same structure as the $V-K$ images, at lower contrast, so we do not present them here. Also, most galaxies reveal very similar morphology in the $J, H$, and $K$ bands, so we only show the $K$ images.

In this section we describe the nuclear morphologies on a galaxyby-galaxy basis, focusing on the information revealed by the $J, H$, and $K$ images. Of course, many of the Toomre-sequence galaxies have also been observed at other wavelengths. For example, seven of the 11 systems have X-ray imaging from either Chandra or XMM-Newton in the X-ray regime, while NGC 6621/6622 was studied with the ROSAT PSPC; only NGC 3509, NGC 7592, and NGC 7764A have not yet been studied in X-rays. Many of the Toomre-sequence galaxies have also been studied with moderate angular resolution in $\mathrm{H}$ I and in the radio continuum and in CO lines (e.g., Norris \& Forbes 1995; Hibbard \& van Gorkom 1996; Georgakakis et al. 2000; Hibbard et al. 2001; Iono et al. 2005). All these observations at other wavelengths have provided a wealth of information. However, we highlight in the discussions below only those aspects that have a direct impact on the interpretation of features observed in the NICMOS images.

One advantage of our high spatial resolution NICMOS data is that it allows better identification of the nuclei in the Toomresequence galaxies than do either ground-based or optical data. In this context we define "nucleus" loosely as a light concentration that has properties consistent with it being the center of the galaxy itself, or one of its merging progenitor galaxies. Table 1 lists the nuclei identified in our data, including their right ascension and declination. These positions are measured from the NICMOS data, and have an absolute accuracy limited to $\sim 1.6^{\prime \prime}$ (Ptak et al. 2006) due to guide-star catalog uncertainties and uncertainties in the relative positions of the HST instruments and Fine Guidance Sensors in the HST focal plane. For the NICMOS imaging of NGC 3921 , we have some evidence that the astrometry is in fact off by several arcseconds, and believe that the absolute positions inferred from the WFPC2 data, as listed in Paper I, are more accurate. While we typically have not marked in Figures 1-16 the positions of the nuclei we identify, their positions can always be easily
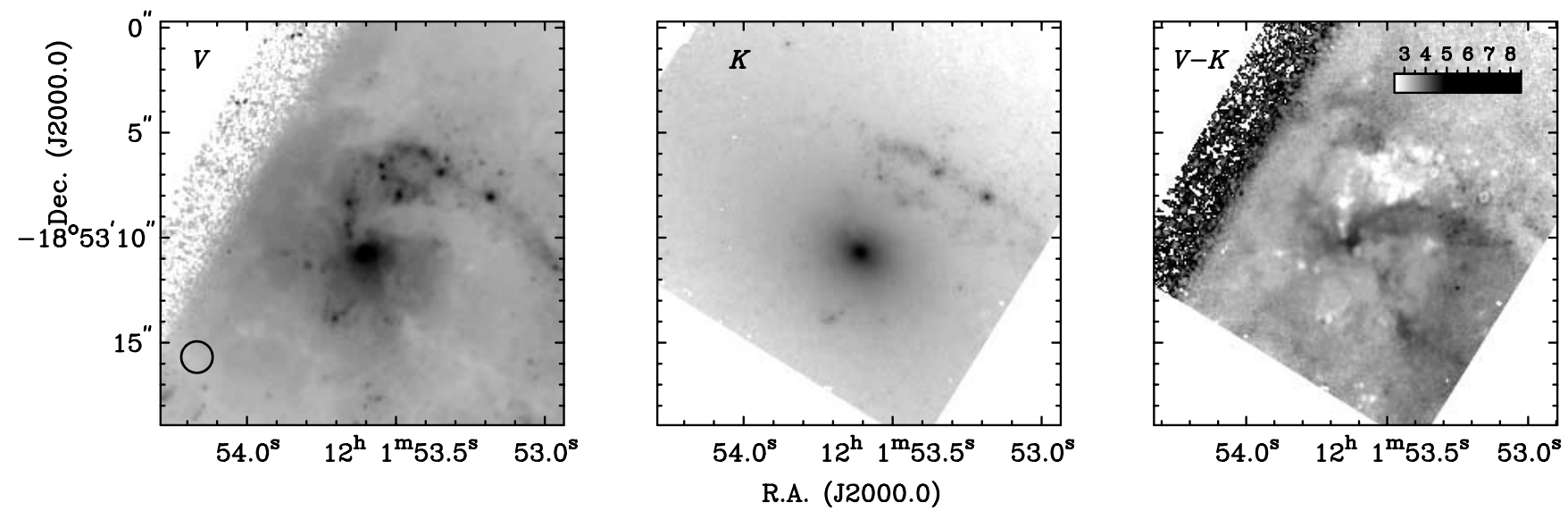

FIG. 2.- Same as Fig. 1, but for NGC 4039 (FOV: $1.3 \mathrm{kpc} \times 1.3 \mathrm{kpc}$ ). 

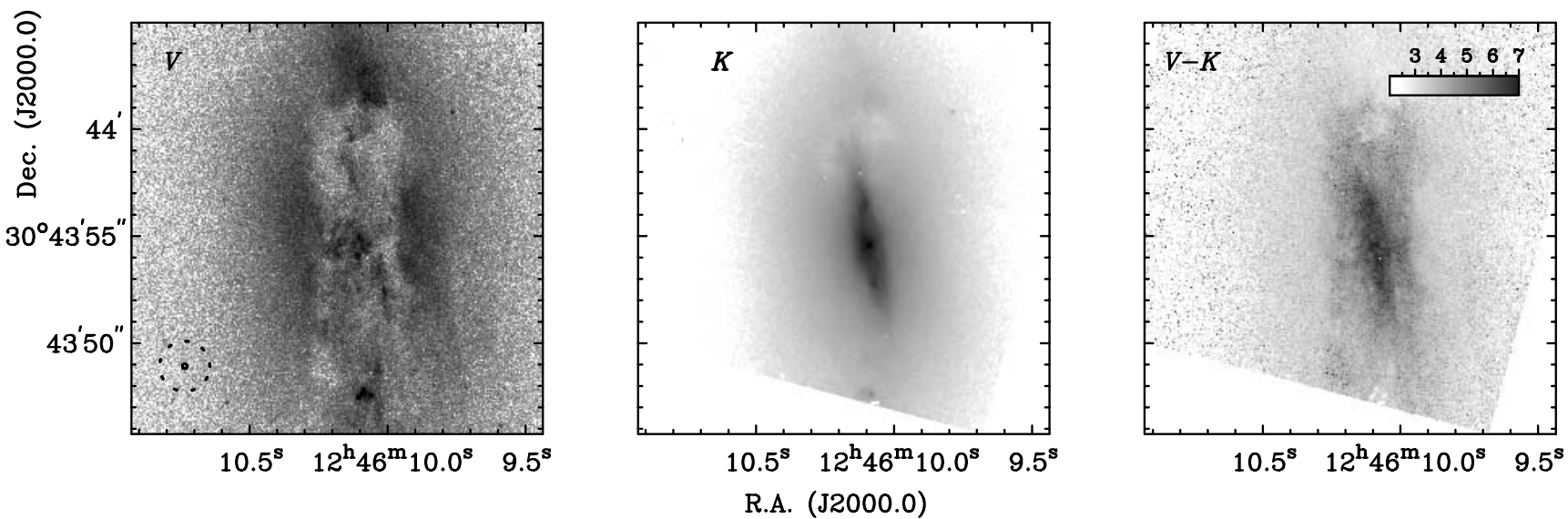

FIG. 3. - Same as Fig. 1, but for NGC 4676A (FOV: $8.1 \mathrm{kpc} \times 8.1 \mathrm{kpc}$ ).

located (if they are not already obvious from the images) by combining the positions in the table with the coordinates listed along the image axes.

In order to unambiguously identify the nuclei of the Toomre sequence, it is important to compare the derived infrared positions of the nuclei with measurements at other wavelengths that are relatively insensitive to dust, such as X-ray, radio continuum, and $\mathrm{CO}$ emission. Table 3 lists the offsets between the absolute positions inferred from our NICMOS data and those inferred from data in these other wavelengths (where available). In general the positions from different wavelengths agree within the uncertainties; in the discussions below we address the nuclear positions only in those cases where the agreement is not good.

Many of the Toomre galaxies have quite complex nuclear morphologies in the NICMOS images. Some interesting features are obvious when blinking or displaying images at various contrast levels on a high-resolution computer screen, but can be much harder to identify and interpret on the figures reproduced here. The discussions presented below are therefore intended to guide the reader through the most relevant features. Descriptions such as "compact," "concentrated," and "extended" are qualitative in nature and refer only to the visual impression obtained from the images. The term "circumnuclear region" is used loosely to denote the region in the immediate vicinity of the nucleus. No particular scale is necessarily implied, but we generally use this term to denote only a small fraction (say, $10 \%-30 \%$ ) of the size of the FOV. The NIC2 FOV corresponds to $7.5 \mathrm{kpc}$ at the median dis- tance of the sample galaxies, so the circumnuclear region does not generally extend beyond $\sim 1 \mathrm{kpc}$ from the nucleus. When referring to the entire NIC2 FOV (which is generally much smaller than the full size of the sample galaxies) we refer to the "central region" of the galaxy. More quantitative measures of the nuclear structure, such as detailed aperture photometry, color index profiles, and surface brightness profiles of the nuclei, are presented and discussed in $\S \S 4$ and 5 .

\subsection{Early-Stage Systems: NGC 4038/4039 (The Antennae), NGC 4676 (The Mice), NGC 7592, and NGC 7764A}

The Antennae ${ }^{10}$ were imaged in two separate pointings, one centered on the nucleus of NGC 4038 (Fig. 1) and the other centered on the nucleus of NGC 4039 (Fig. 2). As a result of the NICMOS aperture location offset discussed in $\S 2.2$, the images were not centered exactly on the presumed nuclear positions (determined from previous WFPC2 imagery). The nucleus of NGC 4039 is an easily identified concentrated source (see Fig. 2). By

\footnotetext{
${ }^{10}$ We use a different distance to the Antennae as compared to Paper I. In that paper we adopted the distance of $21.6 \mathrm{Mpc}$ implied by the observed redshift of the Antennae, neglecting any potential peculiar velocity of the system. Instead, we adopt here the value of $13.8 \mathrm{Mpc}$, which was recently derived from measurements of the tip of the red giant branch (TRGB; Saviane et al. 2004). While this distance puts the Antennae closer than has been assumed in most previous works, the TRGB method has been well demonstrated, and the inferred distance should be more accurate than the canonical redshift distance.
}
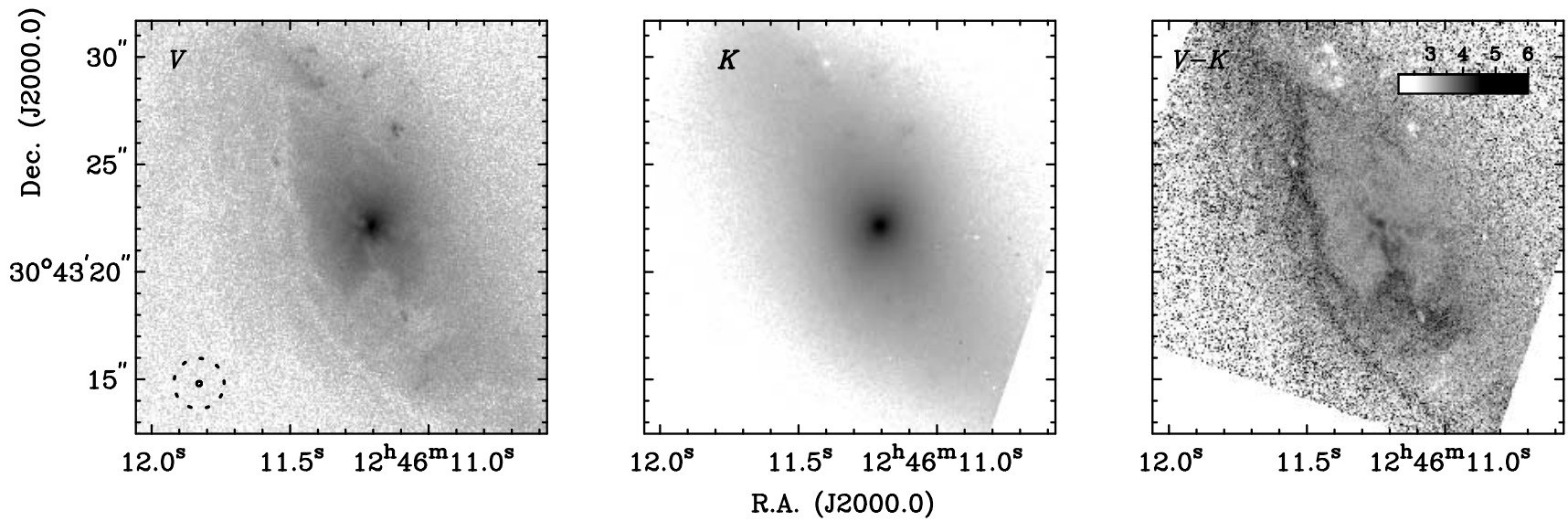

FIG. 4.- Same as Fig. 1, but for NGC 4676B (FOV: $8.1 \mathrm{kpc} \times 8.1 \mathrm{kpc}$ ). 

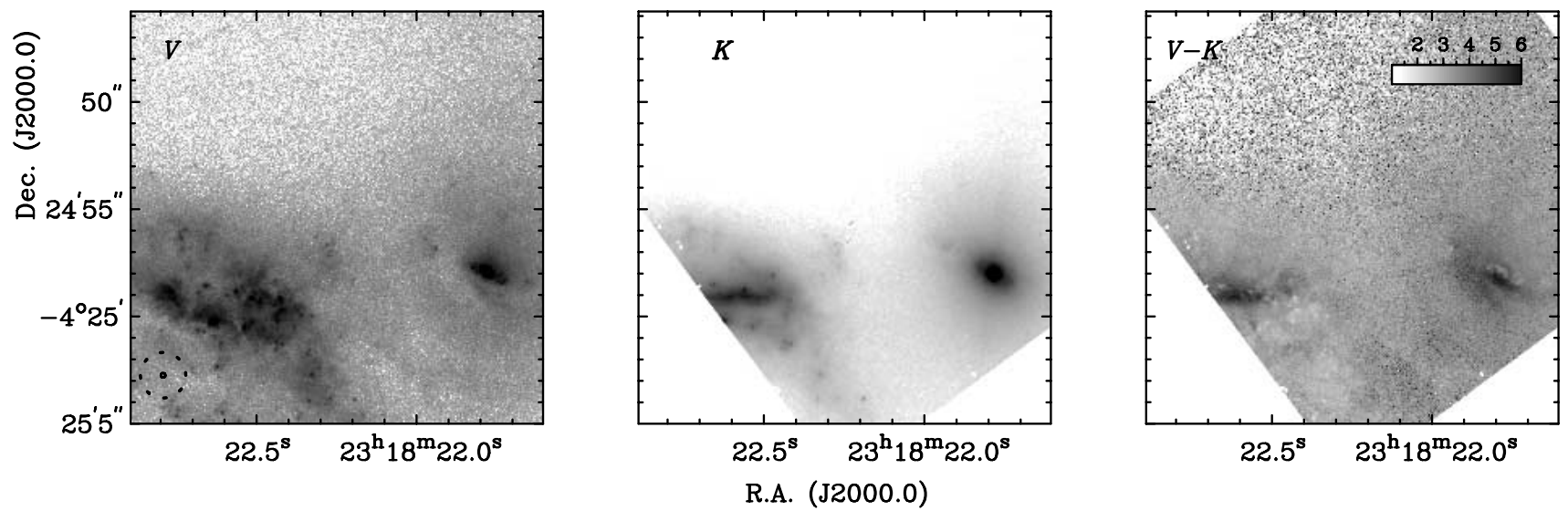

FIG. 5.- Same as Fig. 1, but for NGC 7592 (FOV: $8.9 \mathrm{kpc} \times 8.9 \mathrm{kpc}$ ).

contrast, NGC 4038 does not seem to possess a highly concentrated nuclear region; however, a probable nucleus can be identified in all three NIR filter bands (marked with a square in Fig. 1). Given the patchy dust obscuring the view into the nucleus in the optical regime, optical identifications of the nuclei can be misleading. In the case of NGC 4038, the nucleus identified in Paper I is most likely a circumnuclear $\mathrm{H}$ II region (see also Whitmore et al. 1999). It is very blue in $V-K$, and is likely a foreground starforming knot located in front of a region of high extinction. The nucleus identified here using the NICMOS images is barely visible in the optical.

A comparison with the results obtained from data at other wavelengths does not provide an unambiguous answer to the question of whether the nucleus of NGC 4038 has been correctly identified. This may not be surprising, given the intrinsically complex nature of the nuclear region. We have identified the clump closest to the center of the overall light profile as the nucleus; this position is consistent with that implied by radio continuum data, if one assumes that the nucleus coincides with the brightest source observed at $6 \mathrm{~cm}$ (as assumed for Table 3). However, there are several bright sources in the nuclear area. If the radio continuum nucleus is presumed to reside at the centroid of these sources (as Neff \& Ulvestad 2000 presume; see their Fig. $5 a$ ), then the radio continuum and NICMOS positions are not in mutual agreement. A more accurate alignment of the NIR and radio data might be possible using the technique of Whitmore \& Zhang (2002), but we do not pursue that here.
The Antennae have also been studied extensively in the X-ray regime (e.g., Fabbiano et al. 1997, 2001 and follow-up papers). The NICMOS position of NGC 4038's nucleus is consistent with source 24 in the list of Zezas et al. (2002a, 2002b) and may well be the nucleus (as assumed for Table 3). However, Zezas et al. (2002a, 2002b) actually identify their source 25 as the nucleus, offset in declination by about $6^{\prime \prime}$ from the NICMOS position. There is little evidence in the NICMOS images to favor source 25 as the nucleus, and in fact source 24 has a much larger X-ray flux.

We have observed NGC 4676 in two pointings and clearly detect the nuclei of both galaxies. The northern galaxy, NGC 4676A (Fig. 3), shows a highly concentrated infrared source, completely obscured in the optical, which we identify as the nucleus. The second nucleus (NGC 4676 B) shows a concentrated nuclear region with a smooth appearance (Fig. 4).

We acquired NICMOS images of NGC 7592 in one pointing, as both nuclei in principle fit into the FOV of the NIC2 aperture (see Fig. 5). However, this observation was affected by the NICMOS aperture location offset, discussed in $\S 2.2$, which placed the second circumnuclear region right on the edge of the NIC2 aperture. Although the image covers most of the circumnuclear region, this region shows a very complex morphology, and there is a chance that the true eastern nucleus (NGC $7592 \mathrm{E}$ ) is located outside the FOV. In contrast, the western nucleus (NGC $7592 \mathrm{~W}$ ) is clearly defined and compact, and was previously identified by Rafanelli \& Marziani (1992) to be of Seyfert 2 type.
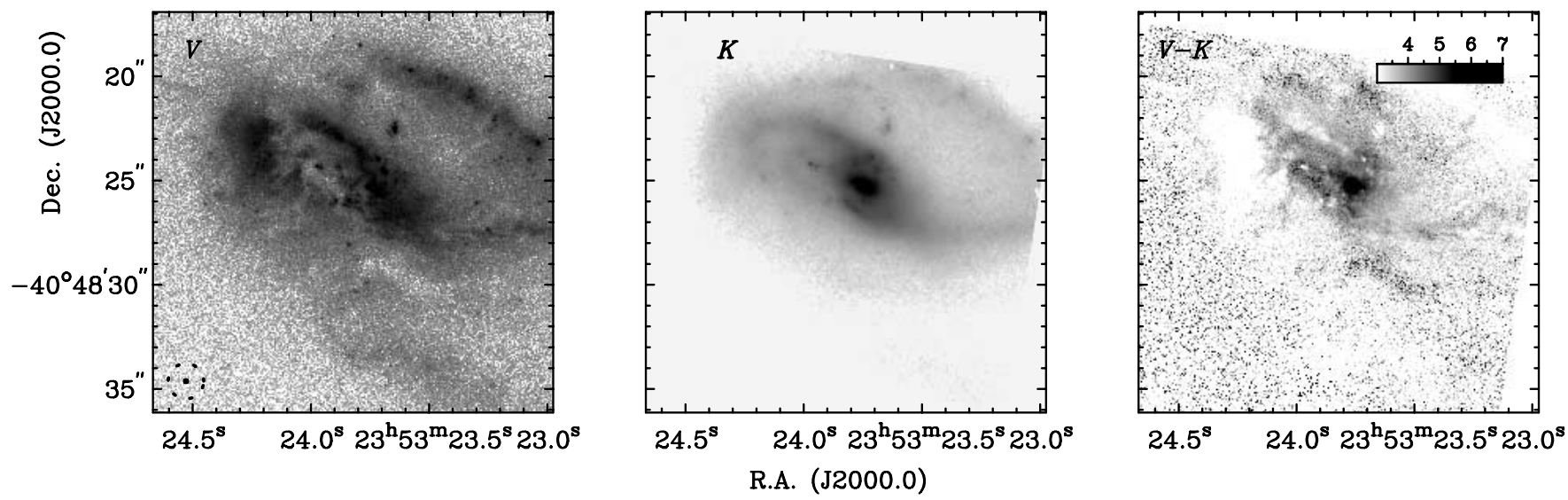

FIG. 6.- Same as Fig. 1, but for NGC 7764A (FOV: $11.3 \mathrm{kpc} \times 11.3 \mathrm{kpc}$ ). 


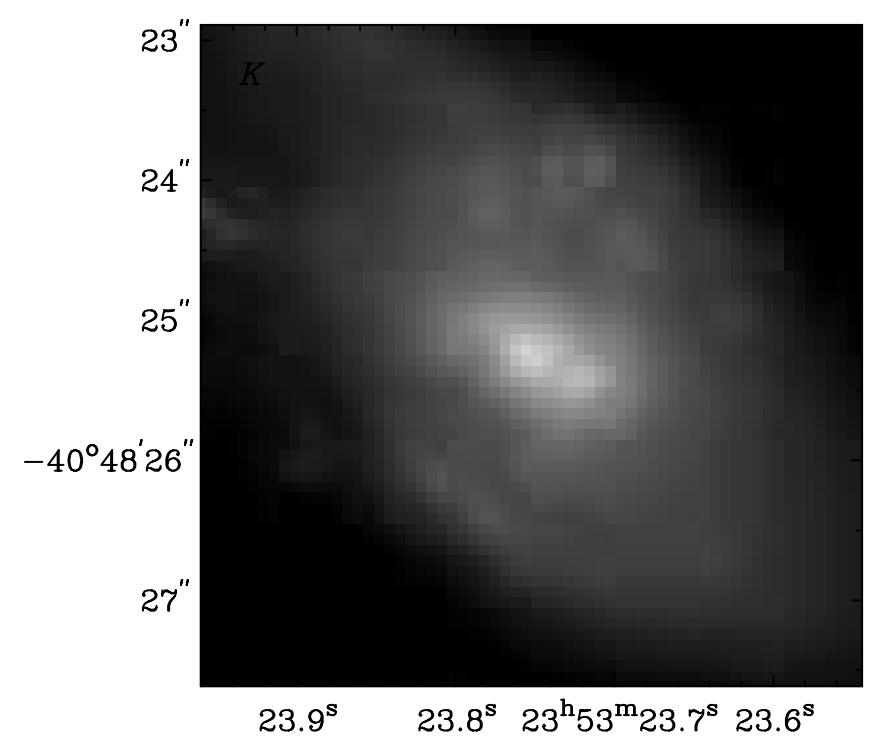

FIG. 7.-Enlarged view of the $K$-band image of the circumnuclear region in NGC 7764A, shown in inverted gray scale and revealing the two nuclei. The displayed FOV translates to a physical size of $2.7 \mathrm{kpc} \times 2.7 \mathrm{kpc}$.

The nuclear region of NGC 7764A is shown in Figure 6. When enlarged, all three NICMOS images $(J, H$, and $K)$ reveal what appears to be a double nucleus in this system (see Fig. 7). If these truly are two merging galaxy nuclei, it is consistent with the placement of NGC 7764A in the middle of the Toomre sequence. The two putative nuclei are separated in a nearly east-west direction by $0.43^{\prime \prime}$, which corresponds to $260 \mathrm{pc}$ at the distance to NGC 7764A. This is similar to the separation of the two nuclei in the merging galaxy Arp 220 (Scoville et al. 1998).

\subsection{Midstage Systems: NGC 6621/6622, NGC 3509, and NGC 520}

The nucleus of NGC 6621 is detected in the infrared and has a fainter counterpart in the optical images (see Fig. 8). The circumnuclear region has a complex morphology, even in the $K$ band. In contrast, the nuclear region of NGC 6622 appears much less complex, showing only a single concentrated source, which we identify as the nucleus (Fig. 9).

The nuclear region of NGC 3509 looks rather inconspicuous, with only a single, well-defined nucleus (Fig. 10), appearing as a concentrated but clearly resolved source. As noted already in
Paper I, NGC 3509 has possibly experienced a minor merger in the past, as only one clear tidal tail is visible in deep optical images (cf. Arp 1966). The relatively low value of the IRAS flux density ratio at $60 \mu \mathrm{m}$ over $100 \mu \mathrm{m}\left(f_{60} / f_{100} \approx 0.33\right)$ also favors a scenario in which star formation has been suppressed compared to the other midstage mergers in the Toomre sequence, which show more vigorous signs of recent or ongoing star formation (cf. Figs. 7 and 8 of Paper I).

NGC 520 was also observed in two pointings. The NIR observations of the southern nucleus (NGC $520 \mathrm{~S}$ ) are quite striking (Fig. 11), revealing a nucleus completely hidden in the optical behind an enormous dust complex. The $J$-band observations show a considerable amount of dust present around the nuclear region. At longer wavelengths ( $H$ and $K$ ) we peer deeper through the dust toward the nucleus, but even the $K$ image is affected by patchy dust structure around the circumnuclear region. However, the nucleus is clearly visible, appearing as an extended disk composed of at least seven major knots. The brightest knot, which we identify as the nucleus, is slightly extended toward the north. Interestingly, the nuclear disk, which appears almost edge-on in the east-west direction, is slightly skewed with respect to the large-scale dust lane. Such a misalignment is also seen in Centaurus A (Schreier et al. 1998).

Previous subarcsecond resolution ground-based NIR imaging also revealed an extended disk around NGC 520 S (Kotilainen et al. 2001). However, considerable substructure was only seen in $\mathrm{Br} \gamma$ observations, not in their $K$-band image. Radio observations at $2 \mathrm{~cm}$ (Carral et al. 1990) also showed an extended disk with five prominent knots, very similar in morphology to our five brightest components. More recently, high spatial resolution radio continuum observations at $21.4 \mathrm{~cm}$ obtained with MERLIN revealed an extended disk with at least nine bright components (Beswick et al. 2003), also consistent with the NIR morphology. The much less obscured northern nucleus (NGC $520 \mathrm{~N}$ ) reveals a bright, centrally confined, compact morphology, embedded in an amorphous disk that is highly inclined to the line of sight (see Fig. 12).

\subsection{Late-Stage Systems: NGC 2623, NGC 3256, $N G C 3921$, and NGC 7252}

The nuclear region of NGC 2623 shows a highly concentrated light profile (Fig. 13). Consistent with our previous findings (see Paper I), only one nucleus is identified, appearing slightly elongated in the east-west direction. There is no clear nucleus discernible in the optical image.
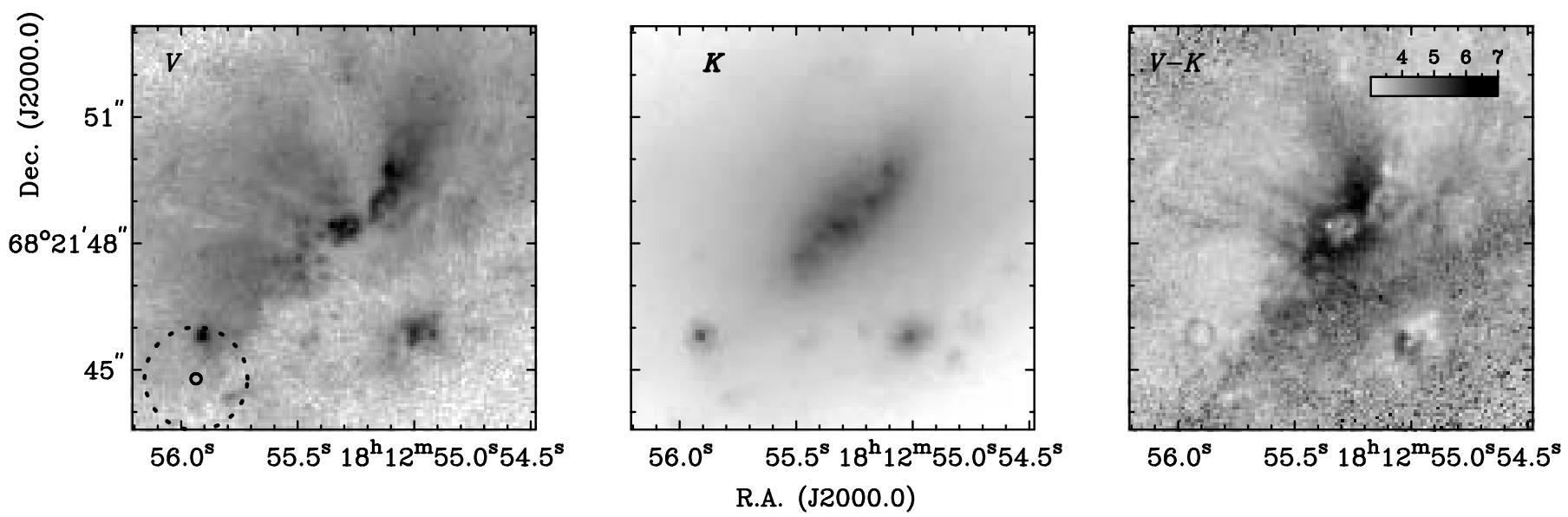

FIG. 8.- Same as Fig. 1, but for NGC 6621 (FOV: $3.9 \mathrm{kpc} \times 3.9 \mathrm{kpc}$ ). 

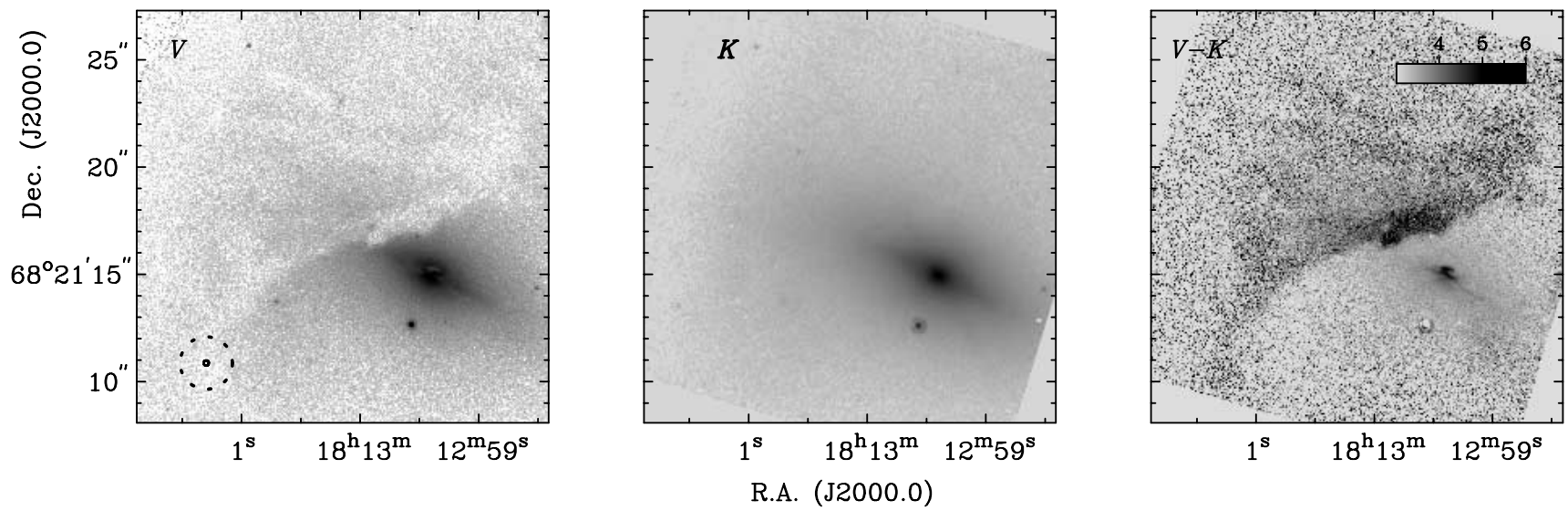

FIG. 9.- Same as Fig. 1, but for NGC 6622 (FOV: $7.8 \mathrm{kpc} \times 7.8 \mathrm{kpc}$ ).

Our analysis of the late-stage merger galaxy NGC 3256 is based on archival NICMOS images in $H$ and F222M (hereafter referred to as $K$; see Fig. 14); no $J$-band data exist. The nuclear region (NGC $3256 \mathrm{~N}$ ) appears as a concentrated and smooth source. Radio imaging at 3 and $6 \mathrm{~cm}$, performed by Norris \& Forbes (1995), identified a second nucleus $5^{\prime \prime}$ south of the primary (NGC $3256 \mathrm{~S})$. Studies of molecular gas emission also hint at a second nucleus at the same location (Sakamoto et al. 2006). Böker et al. (1997) also imaged NGC 3256 in the $N$ band and detected a source at this same position. Based on radio and X-ray power and their ratios, Neff et al. (2003) argue that both nuclei may be low-luminosity active galactic nuclei. In our NICMOS data there is a compact source at the position of the secondary nucleus, but with fainter intensity than the primary nucleus. The source appears projected onto the middle of a spiral arm, lying in a highly reddened region corresponding to a local maximum in the $V-K$ image. It has an extinction-corrected $K$-band magnitude of -18.2 , similar to the nuclei in the other Toomre-sequence galaxies (see $\S 4.3$ ). All this supports the view that there is in fact a highly extincted infrared counterpart to the secondary nucleus that was detected in radio, MIR, and X-ray data.

We also analyzed archival images of NGC 3921. The nuclear region shows a slightly elongated core in the north-northeast to south-southwest direction (see Fig. 15), with only one nucleus detected. Our findings are consistent with earlier studies (Schweizer 1996) and with the fact that NGC 3921 is generally regarded as a late-stage merger remnant with properties resembling elliptical galaxies. In a recent X-ray study by Nolan et al. (2004), however, the X-ray luminosity of the hot diffuse gas was found to be 2 orders of magnitude less than that typical of elliptical galaxies.

Our images of the latest stage merger remnant of the Toomre sequence, NGC 7252, show a nuclear spiral (see Fig. 16), as already detected in the optical and described in our previous study (Paper I). Only one nucleus is identified in all three filter passbands, consistent with previous ground-based studies (e.g., Schweizer 1982; Hibbard et al. 1994) and with the general notion of NGC 7252 being a prototype merger remnant, with an estimated age of about 1 Gyr (e.g., Schweizer 1998).

\section{PHOTOMETRY AND COLORS}

\subsection{Photometry}

We measured the $J, H$, and $K$ magnitudes for all identified Toomre-sequence nuclei within apertures $100 \mathrm{pc}$ and $1 \mathrm{kpc}$ in size. Depending on the distance to each galaxy, the $100 \mathrm{pc}$ aperture corresponds to 2-20 pixels and the $1 \mathrm{kpc}$ aperture corresponds to 23-200 pixels. Fluxes were measured with the IRAF ${ }^{11}$ task phot, centered on the identified nuclei; the resulting magnitudes are listed in Tables 4 and 5. The photometric random errors on the magnitudes and colors are very small (with a median of $0.01 \mathrm{mag}$ ) and are not listed in the tables. The actual errors are dominated by

\footnotetext{
11 IRAF is distributed by the National Optical Astronomy Observatory, which is operated by AURA, Inc., under cooperative agreement with the National Science Foundation.
}
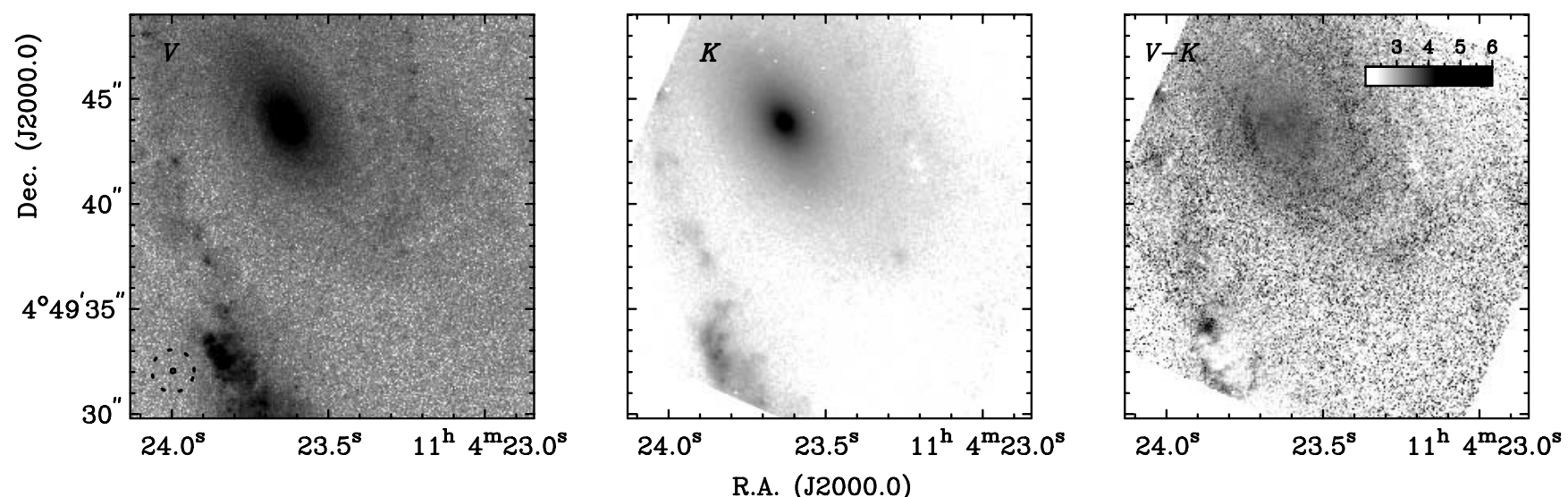

FIG. 10.- Same as Fig. 1, but for NGC 3509 (FOV: $9.5 \mathrm{kpc} \times 9.5 \mathrm{kpc}$ ). 

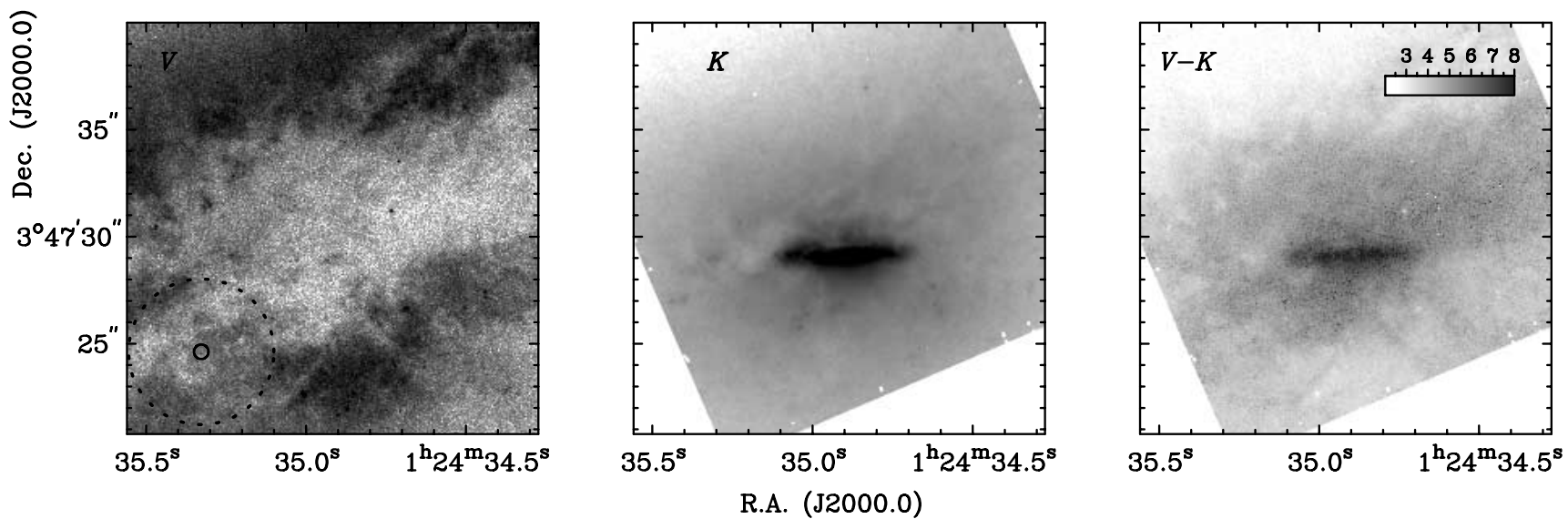

FIG. 11.-Same as Fig. 1, but for NGC $520 \mathrm{~S}$ (FOV: $2.8 \mathrm{kpc} \times 2.8 \mathrm{kpc}$ ).

systematic calibration uncertainties ( $\lesssim 0.05$ mag in each band; see $\S 2.2)$.

\subsection{Near-Infrared Colors}

Figure 17 shows the $J-H$ versus $H-K$ color-color diagram for the $100 \mathrm{pc}$ aperture measurements of the Toomre-sequence nuclei. Separate symbols are used for the early-stage, midstage, and late-stage mergers, as indicated in Table 1. For a direct comparison of the late-stage mergers with elliptical galaxies, we also show in the figure the sample of elliptical galaxies studied by Silva $\&$ Bothun (1998). We transformed their data, which were calibrated to the CIT photometric system, to the Bessell system in which our NICMOS data were calibrated (Bessell \& Brett 1988). We also used the respective $J-H$ and $H-K$ values from the GALAXEV database to generate and overplot the evolutionary track of an aging simple population (Bruzual \& Charlot 2003). We used the Bruzual-Charlot models with the Padua 1994 stellar evolution library for a standard Chabrier initial mass function (IMF) from 0.1 to $100 M_{\odot}$ (Chabrier 2003) and solar metallicity. Two of the three late-stage merger remnants (those which had $J, H$, and $K$ measurements) fall close to the positions of the elliptical galaxies studied by Silva \& Bothun (1998). All Toomresequence nuclei are closely aligned along the reddening vector direction, as expected for dusty stellar populations.

Figure 18 shows the same color-color data for the Toomre nuclei as Figure 17 but focuses on a smaller region of the colorcolor diagram. We compare our data to ground-based measurements from Bushouse \& Stanford (1992; also transformed to the
Bessell system) for a larger sample of interacting galaxies selected from the Arp atlas (Arp 1966). The average and median colors are slightly redder for the Toomre-sequence nuclei than for the Bushouse \& Stanford (1992) galaxies, but this result is only marginally significant given the large scatter in both distributions. Moreover, a direct comparison between the samples is not straightforward. Bushouse \& Stanford (1992) performed photometry in a fixed angular aperture of $5.4^{\prime \prime}$, which is generally much larger than the apertures of a fixed physical size that we have considered for the NICMOS data. Also, Toomre's criteria for characterizing a galaxy as interacting or merging were more stringent than those of Bushouse \& Stanford (1992). In any case, Figure 18 shows that the majority of the Toomre-sequence nuclei fall in the region of the $J-H$ versus $H-K$ diagram that is populated by the sample of Bushouse \& Stanford (1992). The Toomre-sequence nuclei therefore have colors that are comparable to those seen in other interacting and merging galaxies.

In principle it should be possible to constrain the stellar populations in the nuclei of the Toomre-sequence galaxies using the multiband $V, I, J, H$, and $K$ photometry presented here and in Paper I. Such analyses have already been performed for other merging galaxies (e.g., Pasquali et al. 2004). However, in the presence of significant dust, as is the case here, the results are quite uncertain. We therefore postpone a discussion of the stellar populations to a subsequent paper (Paper III), in which we will present HST STIS spectra obtained in the context of our investigation. These spectra can be used to investigate the populations of the nuclei with moderate obscuration. By contrast, NIR spectroscopy
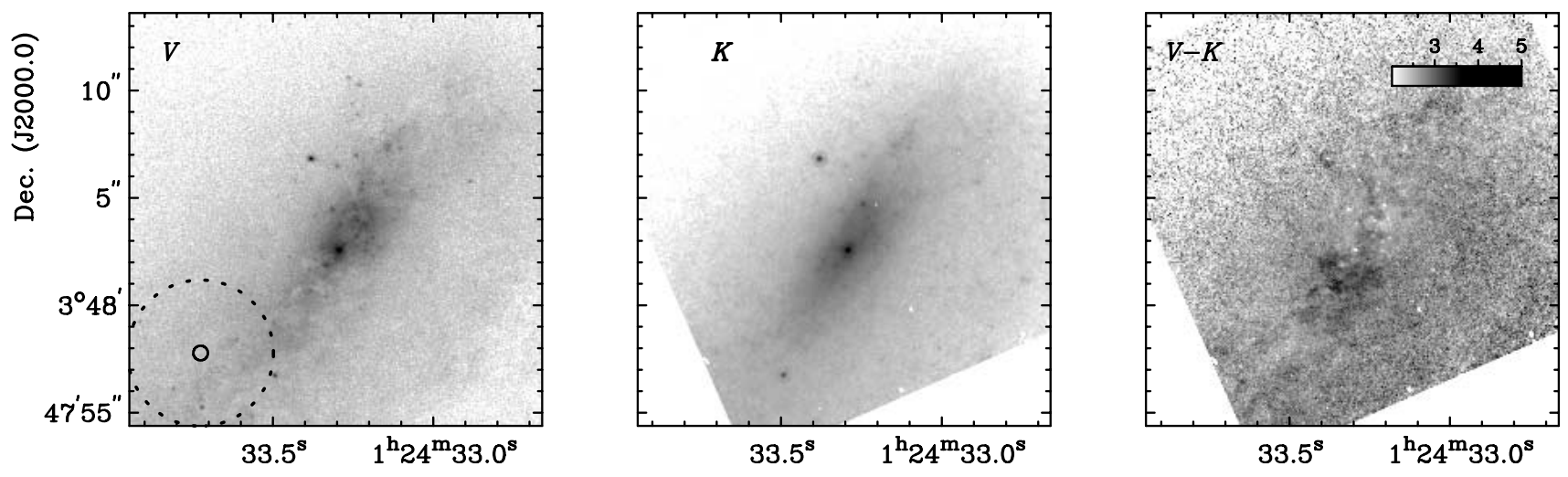

R.A. (J2000.0)

FIg. 12.- Same as Fig. 1, but for NGC 520 N (FOV: $2.8 \mathrm{kpc} \times 2.8 \mathrm{kpc}$ ). 

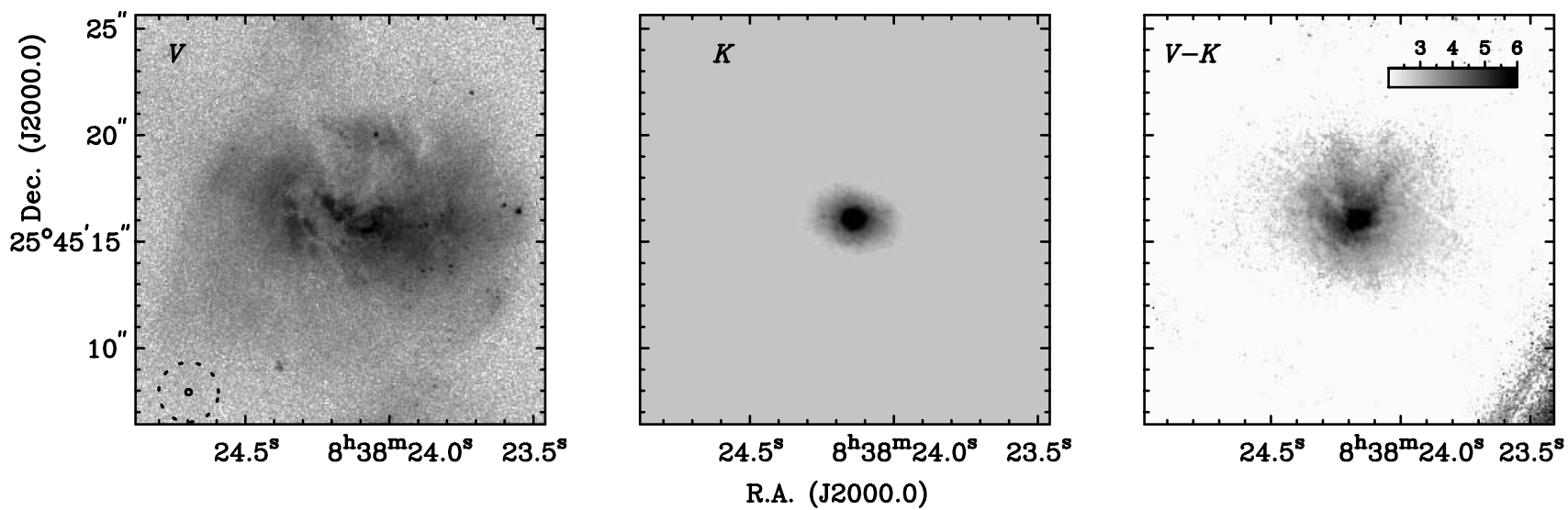

FIG. 13. - Same as Fig. 1, but for NGC 2623 (FOV: $6.8 \mathrm{kpc} \times 6.8 \mathrm{kpc}$ ).

would be required to study the populations of the most heavily obscured nuclei.

\subsection{Dependence on Merger Stage}

In Figures 19 and 20 we examine color trends along the proposed merger sequence. The NIR $J-K$ colors in apertures of $100 \mathrm{pc}$ and $1 \mathrm{kpc}$ are shown as a function of merger stage in Figure 19. The $J-K$ colors vary between 1.0 and 2.1 for all but the two most heavily obscured nuclei (NGC 2623 and NGC 520 S), which have much redder values within the $100 \mathrm{pc}$ and $1 \mathrm{kpc}$ apertures (see Tables 4 and 5, respectively). We have also plotted $V-K$ colors (listed in Tables 4 and 5 ) as a function of merger stage (see Fig. 20), therefore extending the spectral baseline to optical wavelengths. By and large, these yield results consistent with those seen for $J-K$. At the latest stages, the NIR colors tend to be less red and closer to those of normal E/S0 galaxies. Out of 15 nuclei, the three bluest nuclei in a $100 \mathrm{pc}$ aperture are all found in the five latest galaxies of the sequence. While this is suggestive of a trend for the nuclei to become bluer with increasing merger stage, it is clear that there is large scatter in color along the sequence, and that any trend is weak at best and not monotonic.

Unfortunately, the NIR colors measured here do not provide much insight into variations in age along the Toomre sequence, or variations in age between the Toomre-sequence galaxies and elliptical galaxies. The $J-K$ color of a stellar population does not vary much as a function of age. For example, a solar-metallicity population with a Chabrier (2003) IMF has $J-K=0.80,0.57$, 0.82 , and 0.89 , at ages of $10^{7}, 10^{8}, 10^{9}$, and $10^{10} \mathrm{yr}$, respectively (using the Padua 1994 stellar library and an IMF that extends from 0.1 to $100 M_{\odot}$; Bruzual \& Charlot 2003). Moreover, realistic stellar populations for the galaxies in the present sample cannot produce $J-K$ colors that are much redder than $\sim 1.0$. Therefore, the redder $J-K$ colors seen in Figure 19 must be due to dust extinction. As a corollary, the trend that the early-stage mergers are redder than the late-stage mergers merely indicates that the former are dustier than the latter.

Models of merging galaxies with black holes often invoke feedback from an AGN to disperse obscuring dust and shut off star formation (e.g., Hopkins et al. 2005). Combined with the hierarchical formation of structure over a Hubble time, such models can successfully explain a variety of observed properties related to galaxy formation and quasar evolution (e.g., Hopkins et al. 2006). The existence of AGN-driven feedback is supported by observations of individual galaxies such as NGC 6240, which has two AGNs and a strong superwind (Komossa et al. 2003; Gerssen et al. 2004). Our finding that the dust content of the Toomre-sequence nuclei has a decreasing trend with merger stage is qualitatively consistent with the predictions of feedback models. While the Toomre sequence does not currently host many strong AGNs (see Table 1), this is not difficult to reconcile with the feedback models if bright AGN stages are either very short-lived or highly obscured (Hopkins et al. 2005). It will be valuable to use numerical models to make quantitative predictions for the dust and gas content of interacting and merging galaxies as a function of merger (st)age, and compare these to results such as those obtained here.
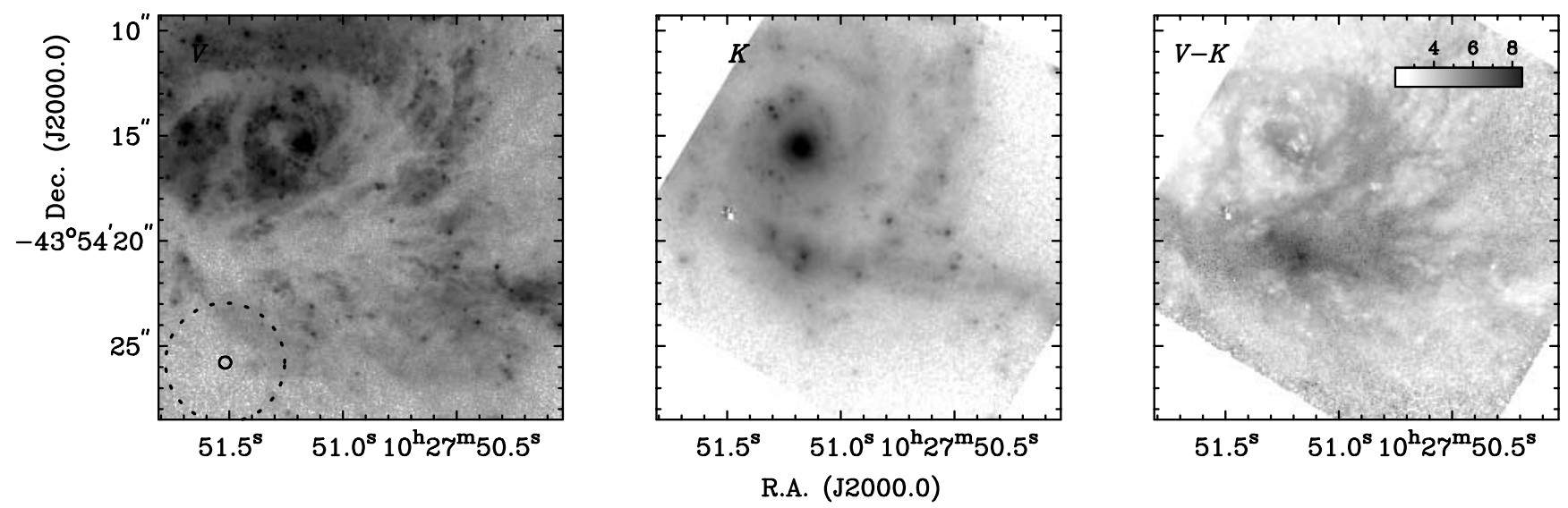

FIG. 14.- Same as Fig. 1, but for NGC 3256 (FOV: $3.4 \mathrm{kpc} \times 3.4 \mathrm{kpc}$ ). 

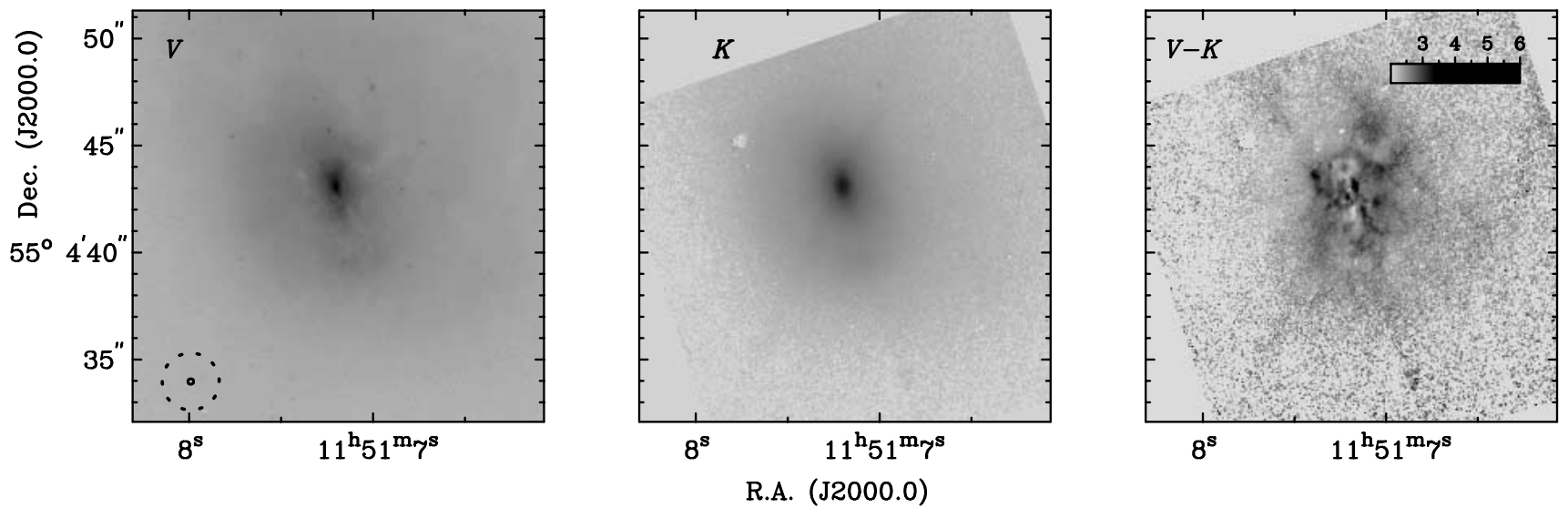

FIG. 15.- Same as Fig. 1, but for NGC 3921 (FOV: $7.2 \mathrm{kpc} \times 7.2 \mathrm{kpc}$ ).

As in our previous WFPC2 study, we also calculate the total luminosity within the $100 \mathrm{pc}$ and $1 \mathrm{kpc}$ apertures for the sample galaxies, in this case for the $K$ band. The $K$-band luminosity $\left(L_{K}\right)$ is shown in Figure 21 as a function of merger stage, where the luminosities have been corrected for dust extinction. To perform this correction, we first assume that the stellar population has an intrinsic $J-K \approx 0.85$. The $K$-band extinction for each nucleus can then be estimated from Tables 4 and 5 using the equation $A_{K}=$ $0.659(J-K-0.85)$, based on the extinction law of Rieke \& Lebofsky (1985). ${ }^{12}$ Our results are not significantly affected for other realistic choices for the intrinsic $J-K$ color. Figure 21 shows that $L_{K}$ (within an aperture of fixed physical size) tends to increase with advancing merger stage. To statistically test this trend, we calculate the Spearman rank-order correlation coefficient $\left(r_{s}\right)$ for each of the $100 \mathrm{pc}$ and $1 \mathrm{kpc}$ data sets. In both cases we find that $r_{s}=0.61$, indicating a $2 \sigma$ correlation $(99 \%$ confidence level [CL]). We note that the Antennae drive much of this correlation; removing the Antennae nuclei from the analysis lowers the correlation coefficient to $r_{s}=0.5$, indicating a $1.5 \sigma$ correlation $(93 \% \mathrm{CL})$. We caution, however, that this analysis assumes that there are no systematic errors which depend on the merger stage. While not shown here, we have done the same analysis for the fractional nuclear luminosity (i.e., $L_{K, \text { nuc }} / L_{K \text {, tot }}$ ) as a function of merger stage and find a similar trend. Because we have corrected for extinction, the underlying cause of this trend must be

${ }^{12}$ For NGC 3256 we used a similar formalism for calculating $A_{K}$ based on the $H-K$ color, because no $J$-band data are available for this galaxy. either an increasing stellar density with advancing merger stage, or a younger average stellar age with advancing merger stage (or both). We cannot discriminate between these two possibilities on the basis of the NIR imaging data alone. Available far-infrared data also cannot address this issue, since the global star formation rates measured by IRAS (e.g., Joseph \& Wright 1985; Casoli et al. 1991) are not necessarily a good indicator of the star formation rates in the nuclei (Kennicutt et al. 1987). As a caveat we would like to note that the observed trend of increasing $L_{K}$ as a function of advancing merger stage is based on the initially assumed merger stage (Toomre 1977), which may not necessarily reflect the actual merger (st)age for all 11 merger systems. Nonetheless, we use the predefined merger stage (which is a fixed value) rather than the merger age. While the very early and very late stages are well defined, an actual ranking (i.e., an exact placement within this sequence) may be possible only after deriving the dynamical history of the merger through detailed simulations.

\subsection{Radial Color Gradients}

The two-dimensional spatial variations of the $V-K$ color in the central regions of Toomre-sequence galaxies are quite complex, as evident from the gray-scale representations in Figures 116. To obtain more easily interpretable quantitative insight, we have therefore determined the radial $V-K$ color gradients for all of the nuclei, measured in concentric circular annuli centered around each nucleus and shown in Figure 22. The annuli are contiguous and grow in radius by a factor of 1.1 at each step. The color profiles are followed out to a distance of $5^{\prime \prime}$ from each nucleus
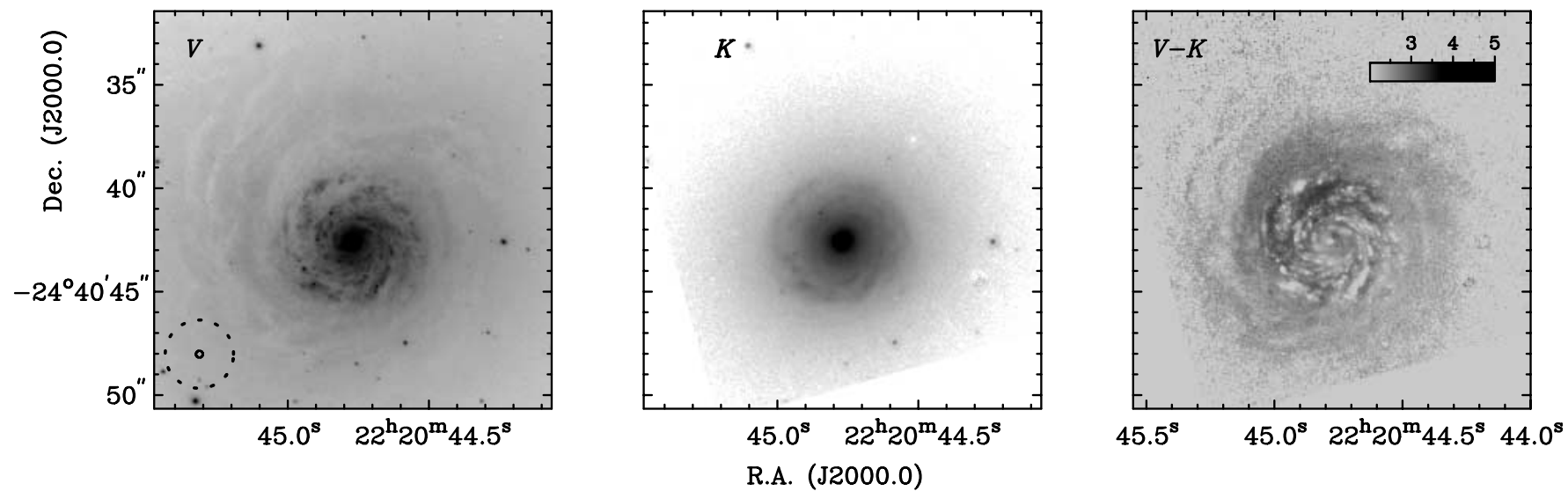

FIG. 16.- Same as Fig. 1, but for NGC 7252 (FOV: $5.8 \mathrm{kpc} \times 5.8 \mathrm{kpc}$ ). 
TABLE 3

Positional Offsets of the Nuclei Compared to Other Wave Bands

\begin{tabular}{|c|c|c|c|c|c|c|c|c|c|}
\hline \multirow[b]{2}{*}{$\begin{array}{c}\text { GaLAXY } \\
\text { (1) }\end{array}$} & \multicolumn{3}{|c|}{ NIR - X-RAY } & \multicolumn{3}{|c|}{ NIR - Radio ContinuUm } & \multicolumn{3}{|c|}{$\mathrm{NIR}-\mathrm{CO}$} \\
\hline & $\begin{array}{c}\Delta \text { R.A. } \\
(\operatorname{arcsec}) \\
(2)\end{array}$ & $\begin{array}{c}\Delta \text { Decl. } \\
(\operatorname{arcsec}) \\
(3)\end{array}$ & $\begin{array}{c}\Delta(\alpha, \delta) \\
(\operatorname{arcsec}) \\
(4)\end{array}$ & $\begin{array}{c}\Delta \text { R.A. } \\
(\operatorname{arcsec}) \\
(5)\end{array}$ & $\begin{array}{c}\Delta \text { Decl. } \\
\text { (arcsec) } \\
(6)\end{array}$ & $\begin{array}{c}\Delta(\alpha, \delta) \\
(\operatorname{arcsec}) \\
(7)\end{array}$ & $\begin{array}{c}\Delta \text { R.A. } \\
(\operatorname{arcsec}) \\
(8)\end{array}$ & $\begin{array}{c}\Delta \text { Decl. } \\
(\operatorname{arcsec}) \\
(9)\end{array}$ & $\begin{array}{c}\Delta(\alpha, \delta) \\
(\operatorname{arcsec}) \\
(10)\end{array}$ \\
\hline NGC $4038 \ldots \ldots \ldots$ & $+1.05^{\mathrm{a}}$ & $+0.42^{\mathrm{a}}$ & 1.8 & $+0.60^{\mathrm{b}}$ & $+1.30^{\mathrm{b}}$ & 1.6 & $\cdots$ & $\ldots$ & $\cdots$ \\
\hline 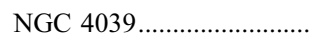 & $+0.11^{\mathrm{a}}$ & $-0.30^{\mathrm{a}}$ & 1.8 & $+1.35^{\mathrm{b}}$ & $+0.30^{\mathrm{b}}$ & 1.6 & $\ldots$ & $\ldots$ & $\ldots$ \\
\hline 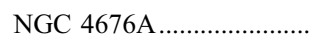 & $0.00^{\mathrm{c}}$ & $-1.20^{\mathrm{c}}$ & 1.8 & $+0.24^{\mathrm{d}}$ & $-0.67^{\mathrm{d}}$ & 1.6 & $+0.75^{\mathrm{e}}$ & $-1.36^{\mathrm{e}}$ & 1.7 \\
\hline 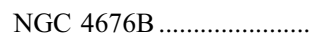 & $-0.30^{\mathrm{c}}$ & $+0.20^{\mathrm{c}}$ & 1.8 & $-1.50^{\mathrm{d}}$ & $-0.86^{\mathrm{d}}$ & 1.6 & $-0.45^{\mathrm{e}}$ & $-1.78^{\mathrm{e}}$ & 1.7 \\
\hline 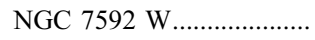 & $\ldots$ & $\ldots$ & $\ldots$ & $+0.90^{\mathrm{f}}$ & $+0.73^{\mathrm{f}}$ & 1.6 & $-0.75^{\mathrm{g}}$ & $+1.20^{\mathrm{g}}$ & 1.6 \\
\hline 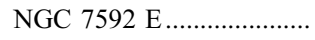 & $\ldots$ & $\ldots$ & $\ldots$ & $\ldots$ & $\ldots$ & $\ldots$ & $\ldots$ & $\ldots$ & $\ldots$ \\
\hline NGC 7764A E ..................... & $\ldots$ & $\ldots$ & $\ldots$ & $\ldots$ & $\ldots$ & $\ldots$ & $\ldots$ & $\ldots$ & $\ldots$ \\
\hline NGC 7764A W .................... & $\ldots$ & $\ldots$ & $\ldots$ & $\ldots$ & $\ldots$ & $\ldots$ & $\ldots$ & $\ldots$ & $\ldots$ \\
\hline NGC $6621 \ldots \ldots \ldots \ldots$ & $\ldots$ & $\ldots$ & $\ldots$ & $+0.60^{\mathrm{h}}$ & $+0.80^{\mathrm{h}}$ & $\leq 2.4$ & $-2.25^{\mathrm{g}}$ & $-0.70^{\mathrm{g}}$ & 1.6 \\
\hline 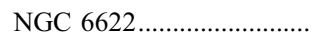 & $\ldots$ & $\ldots$ & $\ldots$ & $\ldots$ & $\ldots$ & $\ldots$ & $\ldots$ & $\ldots$ & $\ldots$ \\
\hline NGC $3509 \ldots \ldots \ldots \ldots \ldots$ & $\ldots$ & $\ldots$ & $\ldots$ & $+0.15^{\mathrm{i}}$ & $+0.80^{\mathrm{i}}$ & $\leq 1.9$ & $\ldots$ & $\ldots$ & $\ldots$ \\
\hline NGC $520 \mathrm{~S}$ & $+4.95^{\mathrm{j}}$ & $+0.70^{\mathrm{j}}$ & $\geq 35.0$ & $+0.15^{\mathrm{k}}$ & $-0.77^{\mathrm{k}}$ & 1.6 & $+1.05^{\mathrm{e}}$ & $-0.70^{\mathrm{e}}$ & $\leq 1.7$ \\
\hline 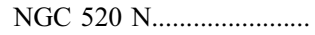 & $\ldots$ & $\ldots$ & $\ldots$ & $\ldots$ & $\ldots$ & $\ldots$ & $\ldots$ & $\ldots$ & $\ldots$ \\
\hline NGC $2623 \ldots \ldots \ldots \ldots \ldots$ & $+0.15^{1}$ & $+2.77^{1}$ & 1.8 & $+0.83^{\mathrm{d}}$ & $-0.59^{\mathrm{d}}$ & 1.6 & $+1.05^{\mathrm{m}}$ & $-0.37^{\mathrm{m}}$ & $\leq 1.7$ \\
\hline 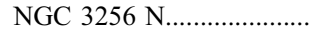 & $-1.20^{\mathrm{n}}$ & $+1.40^{\mathrm{n}}$ & 1.8 & $-0.90^{\circ}$ & $+1.60^{\circ}$ & 1.6 & $\ldots$ & $\ldots$ & $\ldots$ \\
\hline NGC $3256 \mathrm{~S}$ & $-0.90^{\mathrm{n}}$ & $+1.40^{\mathrm{n}}$ & 1.8 & $-0.90^{\circ}$ & $+1.60^{\circ}$ & 1.6 & $\ldots$ & $\ldots$ & $\ldots$ \\
\hline NGC $3921 \ldots \ldots \ldots \ldots$ & $-0.30^{\mathrm{p}}$ & $+1.31^{\mathrm{p}}$ & 2.6 & $+1.11^{\mathrm{d}}$ & $+0.09^{\mathrm{d}}$ & 1.6 & $+0.45^{\mathrm{e}}$ & $+1.57^{\mathrm{e}}$ & $\leq 1.7$ \\
\hline NGC $7252 \ldots \ldots \ldots \ldots$ & $+0.15^{\mathrm{p}}$ & $+1.60^{\mathrm{p}}$ & 2.6 & $+1.20^{\mathrm{q}}$ & $-2.10^{\mathrm{q}}$ & $\leq 2.4$ & $+0.75^{\mathrm{r}}$ & $-0.44^{\mathrm{r}}$ & $\leq 1.9$ \\
\hline
\end{tabular}

Notes.-Col. (1): Galaxy name. Cols. (2) and (3): Positional offsets between the HST and X-ray-detected nucleus. Col. (4): Uncertainty associated with this positional offset. Cols. (5) and (6): Positional offsets between the HST and radio continuum measurements. Col. (7): Uncertainty associated with this positional offset. Cols. (8) and (9): Positional offsets between the HST and CO measurements. Col. (10): Uncertainty associated with this positional offset. The HST positions adopted for the nuclei are the NICMOS positions listed in Table 1, except for NGC 3921, for which we used the WFPC2 position from Paper I (due to evidence for an $\sim 4^{\prime \prime}$ absolute astrometric error in the corresponding NICMOS data set). The sources for the X-ray measurements are Einstein, Chandra, and XMM-Newton, for the radio continuum measurements the VLA, and for the $\mathrm{CO}$ measurements various telescopes. The listed uncertainties associated with the positional offsets are the quadrature sums of the $H S T$ astrometric accuracy and the astrometric accuracies of the observations in the other wave bands. Astrometric accuracies were obtained from the following sources: HST (Ptak et al. 2006), Chandra (Aldcroft 2002; Schade et al. 2002), XMM-Newton and Einstein (S. Snowden 2006, private communication), and the radio continuum and CO (the original literature sources of the data). See the text for a discussion of the X-ray and radio nucleus positions adopted for NGC 4038.

ReFERENCES._- (a) Zezas et al. 2002b; (b) Neff \& Ulvestad 2000; (c) Read 2003; (d) McMahon et al. 2002; (e) Yun \& Hibbard 2001; (f) Condon et al. 1990; (g) D. Iono 2006, private communication; (h) Condon et al. 1996; (i) J. E. Hibbard 2006, unpublished; (j) Fabbiano et al. 1992; (k) Carral et al. 1990; (1) our measurement from archival data; (m) Bryant \& Scoville 1999; (n) Lira et al. 2002; (o) Neff et al. 2003; (p) Nolan et al. 2004; (q) Hibbard et al. 1994; (r) Wang et al. 1992.

TABLE 4

NIR Photometry of the Toomre Sequence Nuclei in 100 pc Apertures

\begin{tabular}{|c|c|c|c|c|c|c|c|}
\hline $\begin{array}{c}\text { Galaxy } \\
\text { (1) }\end{array}$ & $\begin{array}{c}J \\
\text { (mag) } \\
(2)\end{array}$ & $\begin{array}{c}H \\
\text { (mag) } \\
(3)\end{array}$ & $\begin{array}{c}K \\
(\mathrm{mag}) \\
(4)\end{array}$ & $\begin{array}{c}J-H \\
(\mathrm{mag}) \\
(5)\end{array}$ & $\begin{array}{c}J-K \\
(\mathrm{mag}) \\
(6)\end{array}$ & $\begin{array}{c}H-K \\
(\mathrm{mag}) \\
(7)\end{array}$ & $\begin{array}{c}V-K \\
(\mathrm{mag}) \\
(8)\end{array}$ \\
\hline 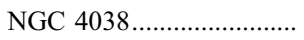 & 16.26 & 15.31 & 14.75 & 0.95 & 1.51 & 0.56 & 4.67 \\
\hline NGC $4039 \ldots \ldots \ldots \ldots$ & 14.62 & 13.67 & 13.42 & 0.95 & 1.20 & 0.25 & 4.50 \\
\hline 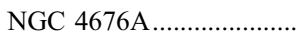 & 18.36 & 16.92 & 16.25 & 1.44 & 2.11 & 0.67 & 6.79 \\
\hline 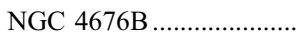 & 17.60 & 16.78 & 16.52 & 0.82 & 1.08 & 0.26 & 3.56 \\
\hline NGC 7592 W...................... & 16.60 & 15.65 & 14.86 & 0.95 & 1.74 & 0.79 & 4.46 \\
\hline NGC 7764A ......................... & 18.25 & 17.14 & 16.61 & 1.11 & 1.64 & 0.53 & 6.05 \\
\hline 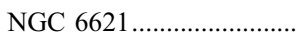 & 17.27 & 16.34 & 15.86 & 0.93 & 1.41 & 0.48 & 4.27 \\
\hline 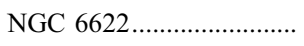 & 16.77 & 15.77 & 15.41 & 1.00 & 1.36 & 0.36 & 4.59 \\
\hline 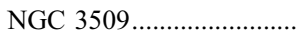 & 17.90 & 17.11 & 16.76 & 0.79 & 1.14 & 0.35 & 3.53 \\
\hline NGC $520 \mathrm{~S}$ & 18.18 & 15.58 & 14.03 & 2.60 & 4.15 & 1.55 & 7.32 \\
\hline 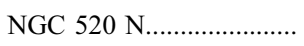 & 16.99 & 16.31 & 15.99 & 0.68 & 1.00 & 0.32 & 2.80 \\
\hline 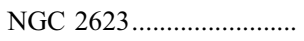 & 16.56 & 14.68 & 13.58 & 1.88 & 2.98 & 1.10 & 8.15 \\
\hline 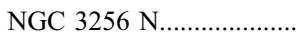 & $\ldots$ & 13.08 & 12.53 & $\ldots$ & $\ldots$ & 0.55 & 4.35 \\
\hline 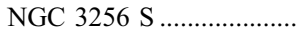 & $\ldots$ & 16.27 & 14.65 & $\ldots$ & $\ldots$ & 1.62 & 7.22 \\
\hline NGC $3921 \ldots \ldots \ldots \ldots$ & 14.71 & 13.97 & 13.70 & 0.74 & 1.01 & 0.27 & 3.39 \\
\hline NGC $7252 \ldots \ldots \ldots \ldots$ & 14.95 & 14.21 & 13.96 & 0.74 & 0.99 & 0.25 & 3.13 \\
\hline
\end{tabular}

Notes.—Col. (1): Galaxy identifier. Cols. (2)-(4): $J$-, $H$-, and $K$-band magnitudes in a 100 pc aperture. Cols. (5)-(8): $J-H, J-K, H-K$, and $V-K$ colors. Note that for NGC 3256 no $J$-band observations were performed. For NGC 7592 and NGC 7764A only the primary nucleus was measured. The photometric random errors on the magnitudes and colors are very small for the NIR bands (with a median of $0.01 \mathrm{mag}$ ) and are not listed in the table. The actual errors are dominated by systematic calibration uncertainties $(\$ 0.05 \mathrm{mag}$ in each band; see $\S 2.2)$. 
TABLE 5

NIR Photometry of the Toomre-Sequence Nuclei in 1 kpc Apertures

\begin{tabular}{|c|c|c|c|c|c|c|c|}
\hline $\begin{array}{l}\text { Galaxy } \\
\text { (1) }\end{array}$ & $\begin{array}{c}J \\
(\mathrm{mag}) \\
(2)\end{array}$ & $\begin{array}{c}H \\
\text { (mag) } \\
(3)\end{array}$ & $\begin{array}{c}K \\
(\mathrm{mag}) \\
(4)\end{array}$ & $\begin{array}{c}J-H \\
(\mathrm{mag}) \\
(5)\end{array}$ & $\begin{array}{c}J-K \\
(\mathrm{mag}) \\
(6)\end{array}$ & $\begin{array}{c}H-K \\
(\mathrm{mag}) \\
(7)\end{array}$ & $\begin{array}{c}V-K \\
(\mathrm{mag}) \\
(8)\end{array}$ \\
\hline 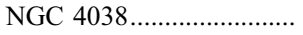 & 12.53 & 11.61 & 10.77 & 0.92 & 1.76 & 0.84 & 3.99 \\
\hline NGC $4039 \ldots \ldots \ldots \ldots$ & 12.55 & 11.77 & 10.96 & 0.78 & 1.59 & 0.81 & 4.24 \\
\hline 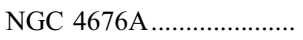 & 14.85 & 13.42 & 12.67 & 1.43 & 2.18 & 0.75 & 5.42 \\
\hline 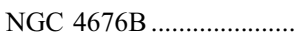 & 14.40 & 13.55 & 13.17 & 0.85 & 1.23 & 0.38 & 3.52 \\
\hline 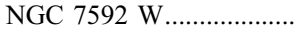 & 14.13 & 13.25 & 12.51 & 0.88 & 1.62 & 0.74 & 4.21 \\
\hline NGC 7764A & 14.99 & 13.88 & 13.22 & 1.11 & 1.77 & 0.66 & 4.55 \\
\hline 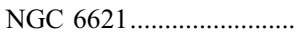 & 13.89 & 12.82 & 12.20 & 1.07 & 1.69 & 0.62 & 4.57 \\
\hline NGC $6622 \ldots \ldots \ldots \ldots \ldots \ldots$ & 13.75 & 12.84 & 12.46 & 0.91 & 1.29 & 0.38 & 3.61 \\
\hline 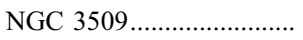 & 14.88 & 14.02 & 13.64 & 0.86 & 1.24 & 0.38 & 3.50 \\
\hline NGC $520 \mathrm{~S}$ & 15.62 & 12.56 & 10.80 & 3.06 & 4.82 & 1.76 & 5.23 \\
\hline NGC 520 N....................... & 13.30 & 13.18 & 12.40 & 0.12 & 0.90 & 0.78 & 2.70 \\
\hline 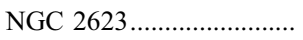 & 13.85 & 12.50 & 11.66 & 1.35 & 2.19 & 0.84 & 3.99 \\
\hline NGC 3256 N...................... & $\ldots$ & 10.93 & 10.16 & $\ldots$ & $\ldots$ & 0.77 & 3.89 \\
\hline NGC $3256 \mathrm{~S}$ & $\ldots$ & 12.18 & 11.02 & $\ldots$ & $\ldots$ & 1.16 & 5.05 \\
\hline 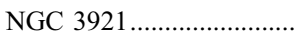 & 12.66 & 11.83 & 11.47 & 0.83 & 1.19 & 0.36 & 3.40 \\
\hline 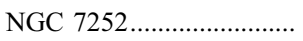 & 12.54 & 11.69 & 11.30 & 0.85 & 1.24 & 0.39 & 3.25 \\
\hline
\end{tabular}

Notes.-Col. (1): Galaxy identifier. Cols. (2)-(4): $J-, H$-, and $K$-band magnitudes in a $1 \mathrm{kpc}$ aperture. Cols. (5)-(8): $J-H, J-K, H-K$, and $V-K$ colors. Note that for NGC 3256 no $J$-band observations were performed. For NGC 7592 and NGC 7764A only the primary nucleus was measured. The photometric random errors on the magnitudes and colors are very small (with a median of $0.01 \mathrm{mag}$ ) and are not listed in the table. The actual errors are dominated by systematic calibration uncertainties ( $\lesssim 0.05$ mag in each band; see $\S 2.2)$.

(which corresponds to between $300 \mathrm{pc}$ and $3.0 \mathrm{kpc}$ for the distances of the sample galaxies). No correction was made for the PSF differences between the $V$ and $K$ bands, so $V-K$ features in the central $\sim 0.2^{\prime \prime}$ should not be trusted.

The last two merger remnants in the sequence (NGC 3921 and NGC 7252) and NGC 3509 all have rather shallow color gradients, with a color change of less than 1 mag within $5^{\prime \prime}$. Most nuclei have steeper gradients, with a change in color of up to 6 mag within $5^{\prime \prime}$.

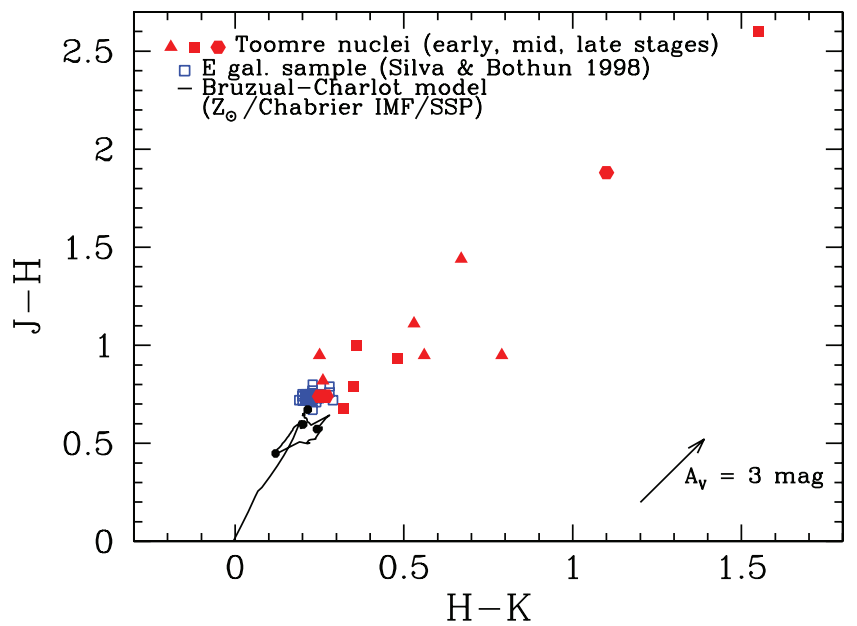

Fig. 17.- $J-H$ vs. $H-K$ diagram of the Toomre nuclei measured within a $100 \mathrm{pc}$ aperture (red symbols). The sample of elliptical galaxies studied by Silva \& Bothun (1998) is also shown (blue open squares). In addition, the BruzualCharlot cluster evolution track for a Chabrier IMF and solar metallicity is overplotted. The black symbols indicate cluster ages of $10^{7}, 10^{8}, 10^{9}$, and $10^{10} \mathrm{yr}$. The indicated reddening vector was calculated from the interstellar extinction law values given by Rieke \& Lebofsky (1985). The random error bars on our measurements are generally smaller than the symbol sizes. The actual errors are dominated by systematic calibration uncertainties ( $\leqslant 0.05 \mathrm{mag}$; see $\S 2.2)$. Note that the symbols for the two late-stage mergers NGC 3921 and NGC 7252 (at $H-K \approx 0.26$ and $J-H=0.74$ ) are partially overlapping.
The nuclei with the strongest color gradients are mostly the midstage mergers. The majority of the nuclei have nuclear colors of $V-K \sim 4-5$, except for the very dusty systems (NGC $520 \mathrm{~S}$, NGC 2623, and NGC 3256 S), which have much redder nuclear colors of $V-K \sim 7-8$ (cf. Fig. 20).

Several of the nuclei become considerably redder inward of about $5^{\prime \prime}$ from the nucleus (e.g., NGC 4676A, NGC 7764A, NGC 520 S, NGC 2623, and NGC 3256 S), probably indicative of a strong concentration of dust in the central regions. This dust

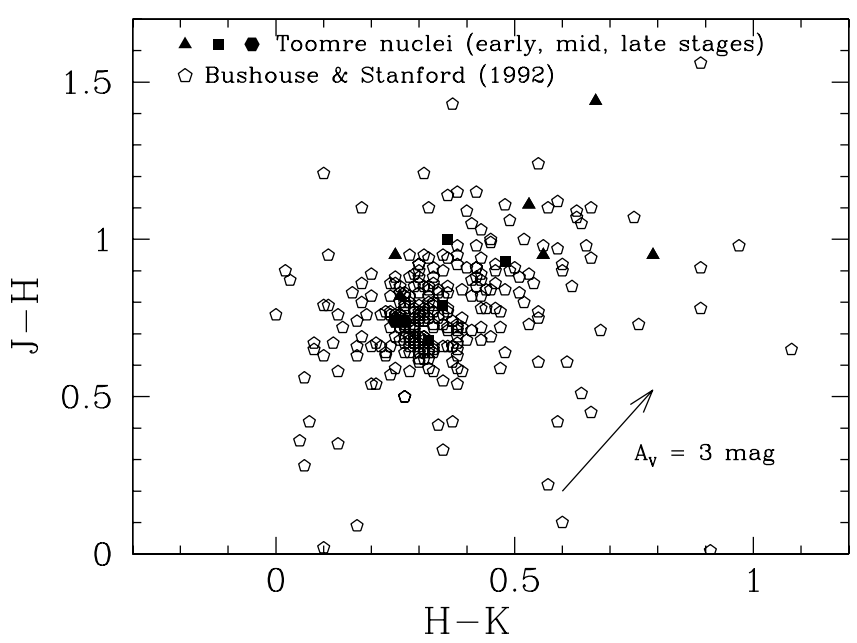

FIG. 18. $-J-H$ vs. $H-K$ diagram of the Toomre nuclei measured within a $100 \mathrm{pc}$ aperture. The merger sample studied by Bushouse \& Stanford (1992) is overplotted (open symbols). Note that we have plotted this diagram only to $J-H=1.7$, omitting the two nuclei with the reddest colors (NGC $520 \mathrm{~S}$ and NGC 2623; see Fig. 17). The indicated reddening vector was calculated from the interstellar extinction law values given by Rieke \& Lebofsky (1985). The random error bars on our measurements are generally smaller than the symbol sizes. The actual errors are dominated by systematic calibration uncertainties $(\lesssim 0.05$ mag; see $\S 2.2$ ). Note that the symbols for the two late-stage mergers NGC 3921 and NGC 7252 (at $H-K \approx 0.26$ and $J-H=0.74$ ) are partially overlapping. 


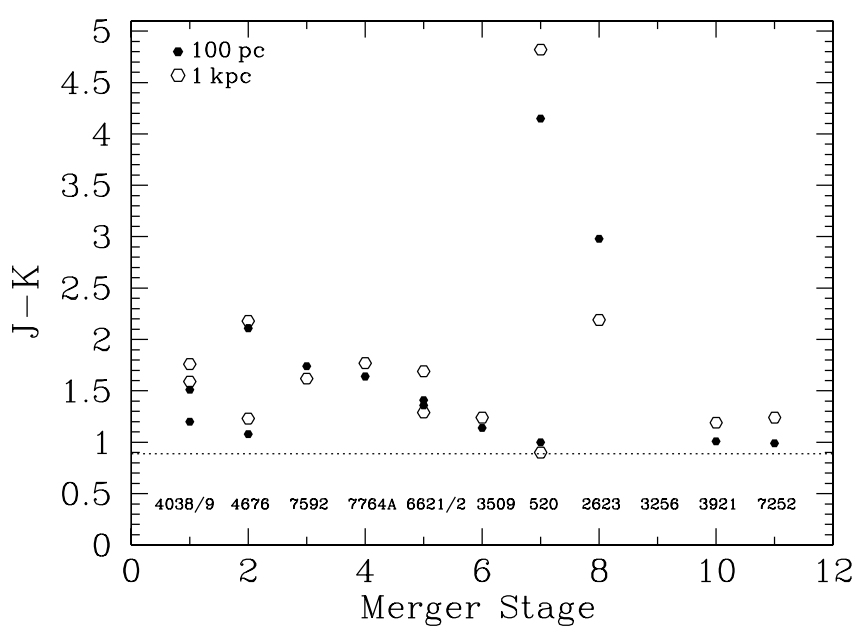

FIG. 19.- $J-K$ color measured within $100 \mathrm{pc}$ ( filled symbols) and $1 \mathrm{kpc}$ (open symbols) apertures as a function of the Toomre-sequence merger stage. The random uncertainties in the colors are generally smaller than the symbol sizes. The true uncertainties in the colors are dominated by systematic uncertainties in the absolute photometric calibration (see $\S 2.2$ ). The dotted horizontal line indicates the $J-K$ color for a $10^{10}$ yr population of solar metallicity (Bruzual \& Charlot 2003), which provides a reasonable fit to the actual colors measured for $\mathrm{E} / \mathrm{S} 0$ galaxies. Note that for NGC 3256 there are no $J$-band measurements available.

complicates the identification of potential population gradients from the observed color gradients. NGC 6621 becomes bluer in its central $\sim 0.6^{\prime \prime}$, but the morphology of the $V-K$ image (see Fig. 8) suggests that this may be due to an absence of dust rather than the presence of young stars. A few nuclei are bluer in their central $\sim 0.2^{\prime \prime}$ (e.g., NGC $520 \mathrm{~N}$ and NGC 7252), but this may be due to PSF differences between $V$ and $K$ rather than differences in stellar populations. On the whole, Figure 22 certainly shows no evidence for the bluer nuclear colors that might be expected if the galaxies all had recent nuclear star formation. On the other hand, this scenario is certainly not ruled out by the data, given that large quantities of dust affect most of the color gradients.

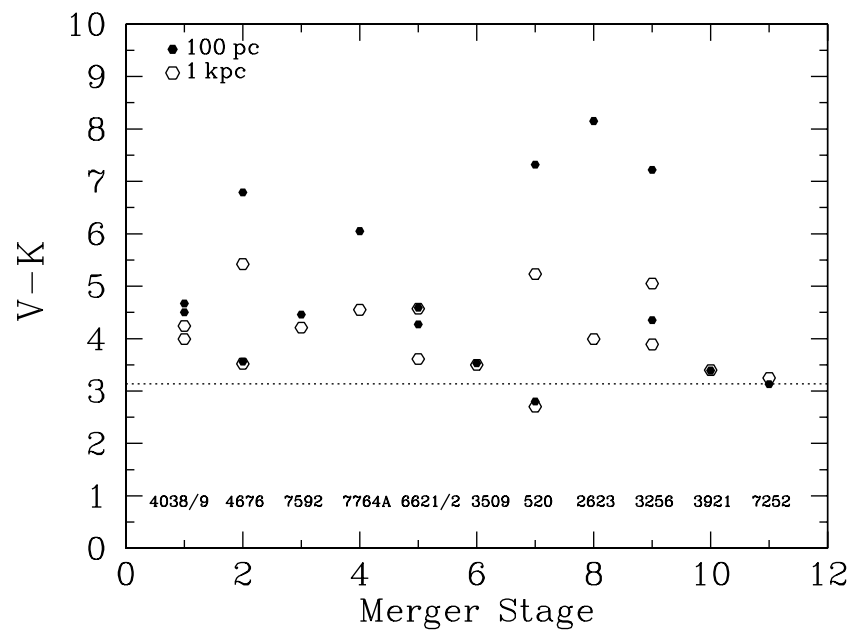

Fig. 20. $-V-K$ color measured within $100 \mathrm{pc}$ ( filled symbols) and $1 \mathrm{kpc}$ (open symbols) apertures as a function of the Toomre-sequence merger stage. The random uncertainties in the colors are generally smaller than the symbol sizes. The true uncertainties in the colors are often dominated by systematic uncertainties in the absolute photometric calibration (see $\S 2.2$ ). The dotted horizontal line indicates the $V-K$ color for a $10^{10} \mathrm{yr}$ population of solar metallicity (Bruzual \& Charlot 2003), which provides a reasonable fit to the actual colors measured for $\mathrm{E} / \mathrm{S} 0$ galaxies.

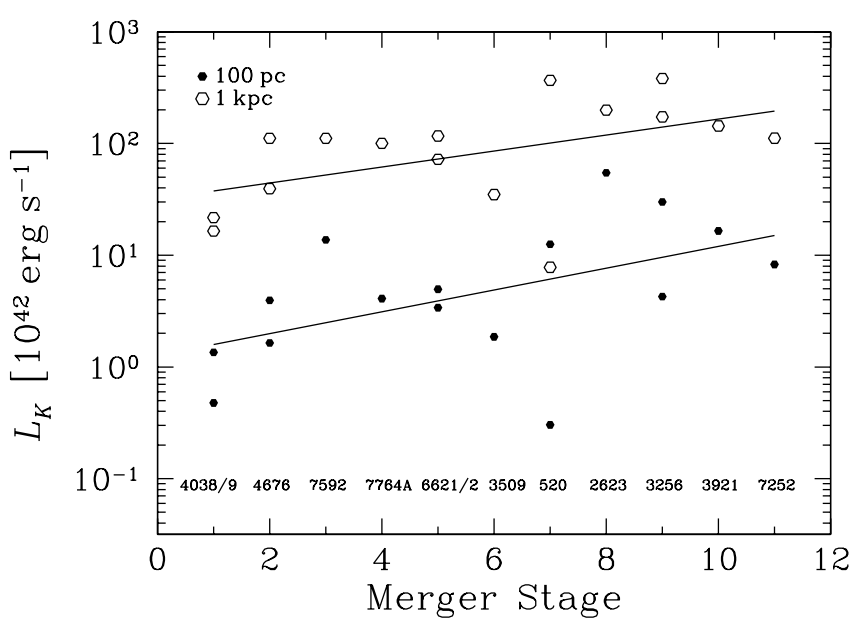

Fig. 21. $-K$-band luminosity (corrected for dust extinction) within a $100 \mathrm{pc}$ ( filled symbols) and $1 \mathrm{kpc}$ (open symbols) aperture as a function of merger stage. The luminosities were corrected for dust extinction based on the observed $J-K$ color, as described in the text. The random uncertainties in the luminosities are generally smaller than the symbol sizes. The true uncertainties are dominated by uncertainties in the galaxy distances. The solid lines are linear least-squares fits intended to guide the eye. There is a trend of increasing nuclear $K$-band luminosity as a function of merger stage.

\section{SURFACE BRIGHTNESS PROFILES}

\subsection{Analysis}

Surface brightness profiles for the Toomre nuclei were derived primarily from the $K$-band imaging, as they are least affected by dust and star formation. The images were run through 20 iterations of Lucy-Richardson deconvolution (Richardson 1972; Lucy 1974) using the VISTA software package (Lauer 1986), where the use of a higher number of iterations did not result in a significantly different deconvolved image. We used TinyTim (Krist \& Hook 2001) to generate a PSF for each nucleus, modeled at the specific position of each nucleus on the detector array and using a K-type star for the PSF spectral distribution. For NGC 2623 we used drizzled images (Scoville et al. 2000) with half the pixel size of the original data. In each case, the PSF was generated out to a radius of $3^{\prime \prime}$, which incorporates the low-level emission in the extended wings, and was tapered at the edges with a Gaussian taper. A few of the galaxies have nuclei that are compact enough to produce a strong Airy ring and diffraction spike pattern. We found that these artifacts were in general successfully mitigated by our deconvolution procedure, although some residuals do remain (especially for the most compact sources, such as NGC 2623).

The nuclear positions were determined by finding the centroid in a small square, typically with a size of about $7 \times 7$ pixels. We then fitted ellipses to the isophotes of the two-dimensional convolved and deconvolved images. The surface brightness profile was derived only for the nuclear region (usually out to a radius of a few arcseconds). We did not attempt to mask out dust or stellar clusters within the area where the surface brightness profile was derived, since the effects of dust are subtle in most cases and there were usually no strong point sources within the few-arcsecond radius that could have interfered with the profile. In cases where such sources exist (e.g., NGC 6621), the profile was only extracted within the innermost nuclear area. The effects of subtle dust features and circumnuclear spiral arms are still visible as small-scale wiggles in some of the luminosity profiles (e.g., NGC 3256 and NGC 7252).

Following closely the procedure used by Laine et al. (2003a) for radii smaller than $0.5^{\prime \prime}$, we used the VISTA profile task (Lauer 

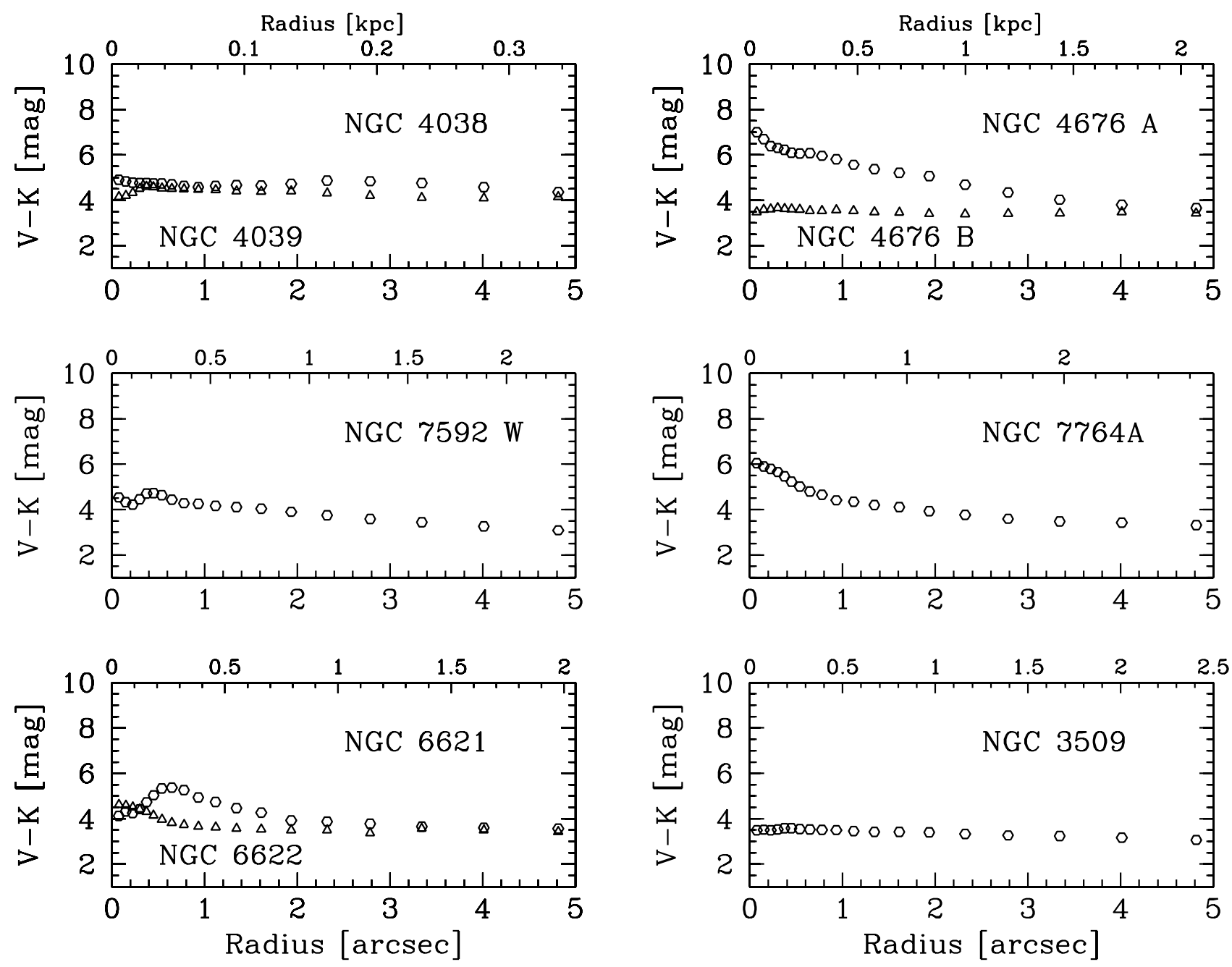

FIG. 22.--Radial $V-K$ color gradients for the regions around each of the Toomre-sequence nuclei, measured using circular annuli. The results for different nuclei in the same interacting system are shown using different symbols in one panel, as labeled. Note that no corrections were made for differences in the PSF FWHM between the $V$ and $K$ bands, leading to spurious artifacts at radii smaller than $0.2^{\prime \prime}$. The $x$-axis measures radius in both arcseconds at the bottom and kiloparsecs at the top.

1985) on the deconvolved image to find the surface brightness profile. This task keeps the center fixed and fits ellipses by sampling the light profile in a circle with a radius of $1,2,3$, etc. pixels. The snuc task (Lauer 1986) in VISTA was used on the deconvolved image between the radii of $0.5^{\prime \prime}$ and $1^{\prime \prime}$ to obtain the surface brightness profile. Outside of $1^{\prime \prime}$ the effects of the PSF are negligible (although diffraction spikes from a very compact nucleus may still be visible there). At these radii we therefore used the snuc task on the surface brightness profile inferred from the original (not PSF-deconvolved) images, motivated by S/N considerations. The transition at $0.5^{\prime \prime}$ was based on experience, which indicated that the profile task is more robust in the interpolation of pixels at very small radii (T. Lauer 2005, private communication). The results of the analysis are shown in Figure 23 for 12 Toomresequence nuclei. The remaining four nuclei (NGC 4038, NGC $7592 \mathrm{E}$, NGC 7764A, and NGC $520 \mathrm{~S}$ ) were too complex or otherwise unsuitable for ellipse fitting and profile extraction. The first panel shows for comparison the profile of the NICMOS PSF itself, indicated by star symbols. Figure 23 also shows for each galaxy the ellipticity and position angle of the fitted ellipses, which can potentially be used to trace structures such as nuclear bars and spiral arms. The random uncertainties for all profiles are usually smaller than the size of the plotted symbols and hence are not shown.
A common analytic function to fit to the luminosity surface density $\Sigma$ of elliptical galaxies is the so-called Nuker law (e.g., Lauer et al. 1995; Byun et al. 1996):

$$
\Sigma(r)=\Sigma_{b} 2^{(\beta-\gamma) / \alpha}\left(r / r_{b}\right)^{-\gamma}\left[1+\left(r / r_{b}\right)^{\alpha}\right]^{(\gamma-\beta) / \alpha} .
$$

The surface brightness $\mu$ follows from

$$
\mu\left[\operatorname{mag} \operatorname{arcsec}^{-2}\right]=-2.5 \log \Sigma\left[L_{\odot} \mathrm{pc}^{-2}\right]+21.572+M_{K, \odot},
$$

where the solar absolute magnitude in the $K$ band is $M_{K, \odot}=$ 3.28 (Binney \& Merrifield 1998). The Nuker law represents a power law with a break at the radius $r_{b}$, with the parameter $\alpha$ measuring the sharpness of the break. The asymptotic power-law slope is $\gamma$ at small radii and $\beta$ at large radii, while $\Sigma_{b}$ is the luminosity surface density at $r_{b}$. The best-fitting Nuker laws are plotted in Figure 23 as solid curves, and the fit parameters are listed in Table 6 , where $\mu_{b}$ is derived from the fitted parameter $\Sigma_{b}$.

As a diagnostic in the discussion below we generally use the quantity $\Gamma_{0.15}$, defined as the power-law slope at a fixed, resolved radius of $0.15^{\prime \prime}$. This is a slightly more robust measure of the central brightness profile slope than the fit parameter $\gamma$, although the 

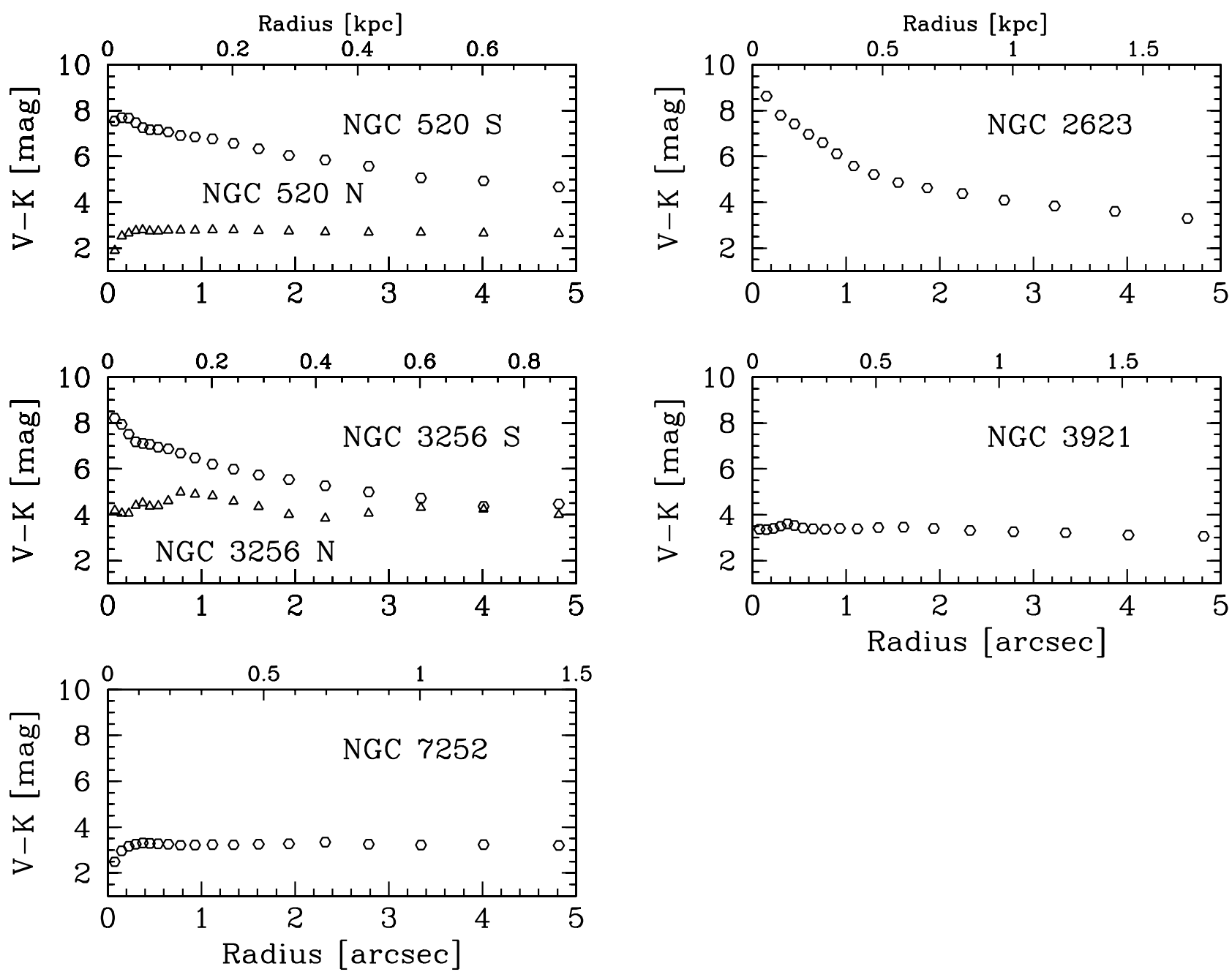

FIG. 22-Continued

two are generally in good agreement (Laine et al. 2003a). Both quantities are listed in Table 6 . Galaxies with slope $\gtrsim 0.5$ are traditionally called power-law galaxies, and those with slope $\lesssim 0.3$ are traditionally called core galaxies (Faber et al. 1997). Whether these values bracket truly distinct classes of galaxies or whether there is simply a continuum in cusp slopes among galaxies continues to be debated (R01; Rest et al. 2001; Lauer et al. 2005; Ferrarese et al. 2006). Here we merely adopt the accepted terminology without necessarily implying that galaxies come in two physically distinct classes.

As a consistency check we also derive surface brightness profiles for a few galaxies from the $J$ - and $H$-band images, yielding very similar results to the $K$ band (apart from an overall color difference). As the $J$ - and $H$-band data have a somewhat narrower PSF than the $K$-band data, the good agreement therefore indicates that uncertainties in the $K$-band surface brightness profiles due to residual problems associated with PSF deconvolution are small. In the remainder of the paper we discuss only the surface brightness profiles derived from the $K$-band images.

\subsection{Individual Galaxy Profiles}

The profiles for the nuclei NGC 3256 N, NGC 3509, NGC 4039, NGC 4676B, NGC 7252, and NGC 7592 W are essentially pure power laws with an almost constant slope through the fitted region. The $\Gamma_{0.15}$ values vary between 0.94 and 1.58 , therefore belonging comfortably to the regime of power-law galaxies. Some deviations from the best fit are seen in the profiles of NGC 3256, NGC 7252, and NGC 7592, most likely due to intrinsic structure in these nuclei. The first two of these galaxies have circumnuclear spiral structure, whereas NGC 7592 has an end-brightened barlike structure. In all cases, residual structure from the diffraction pattern may play some role as well. Either way, in all three cases the overall slope remains relatively unchanged from large to small radii within the fitting area.

The $\Gamma_{0.15}$ values of NGC 2623, NGC 3921, NGC 4676A, and NGC 6621 are large enough to place them in the realm of powerlaw galaxies. However, the surface brightness profiles of these galaxies do show more structure than a pure power law. NGC 2623, for example, shows several wiggles in its profile. Nonetheless, the brightness profile is steep throughout the entire nuclear region, with $\Gamma_{0.15}=1.42$ and no break toward a shallow core. The profile of NGC 3921 appears to have a shallow break around $0.25^{\prime \prime}$, but with an inner slope $\left(\Gamma_{0.15}=1.13\right)$ that is still in the powerlaw regime. NGC $4676 \mathrm{~A}$ has a clearer break in the power-law slope, around $0.43^{\prime \prime}$, but its $\Gamma_{0.15}$ value of 0.65 also still places it comfortably among power-law galaxies. Some curvature around $0.4^{\prime \prime}$ is seen in the profile of NGC 6621, but the $\Gamma_{0.15}$ value of 0.68 indicates that this nucleus too belongs to the power-law group.

NGC 6622 is the only galaxy for which the profile turns over to a power-law slope $\gamma=0.24$ that is characteristic of core galaxies. However, the turnover happens inside $0.1^{\prime \prime}$, the characteristic resolution of our data. So while this galaxy may have a core-type 


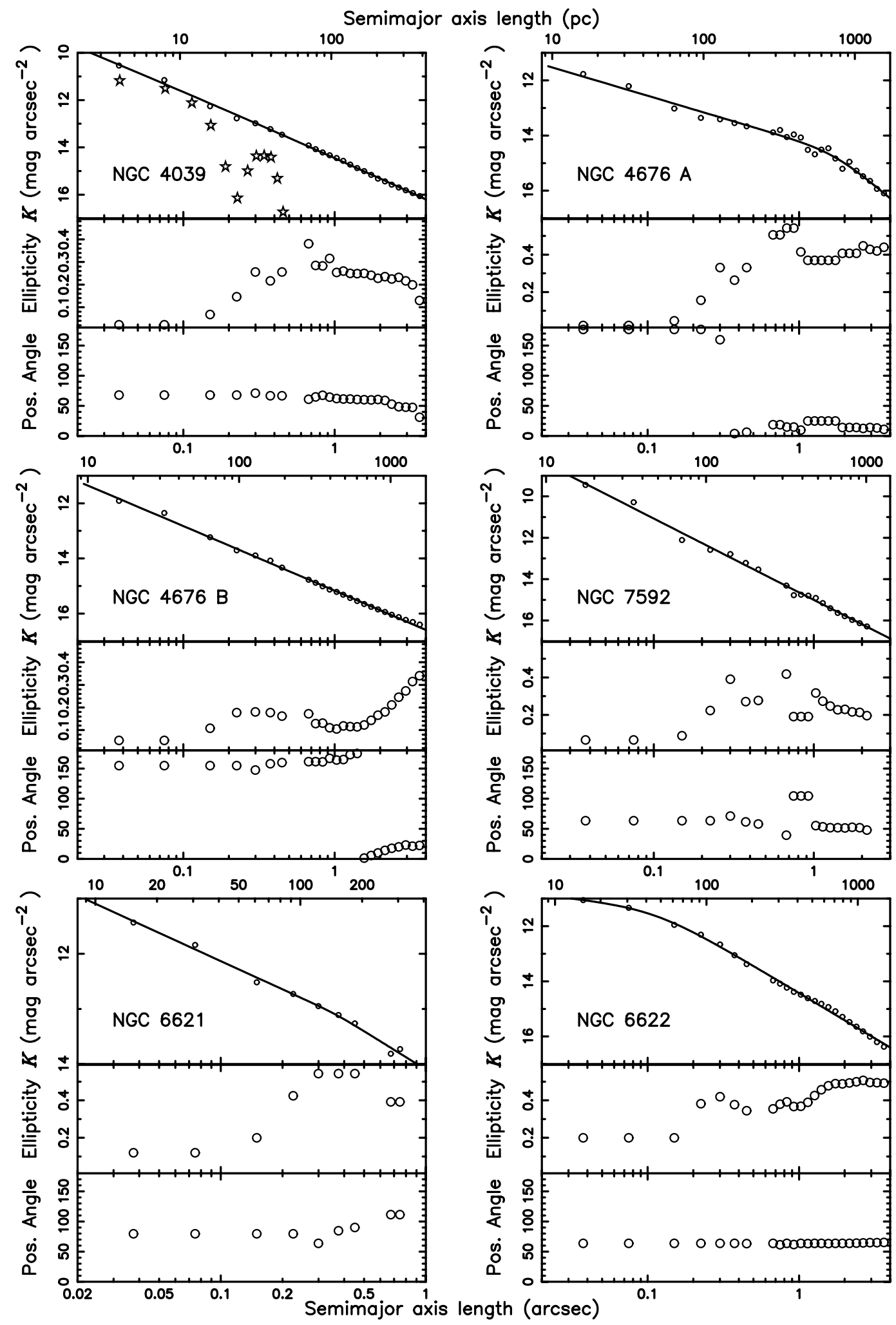

Fig. 23. - Major-axis surface brightness profiles of the nuclear regions of selected Toomre-sequence galaxies, where a fit was possible. The subpanels show the surface brightness profiles overplotted with a Nuker model fit (solid line). The host galaxies of the individual nuclei are labeled. For comparison, we plot the profile of the NICMOS PSF in the first panel (star symbols, arbitrarily normalized).

profile, it is certainly not an unambiguous case. In the following we do not discuss the parameter $r_{b}$ of the Nuker profile fits. This parameter generally has physical meaning only for galaxies with well-resolved cores, and none of the Toomre-sequence nuclei fall in this category.
The only nucleus that does not fit within the class of either the core or the power-law galaxies is one nucleus in a midstage merger, NGC $520 \mathrm{~N}$. This nucleus has a very shallow profile out to several arcseconds from the center, but with a strong upturn in the central $\sim 0.15^{\prime \prime}$. The bright central source responsible for this upturn is 

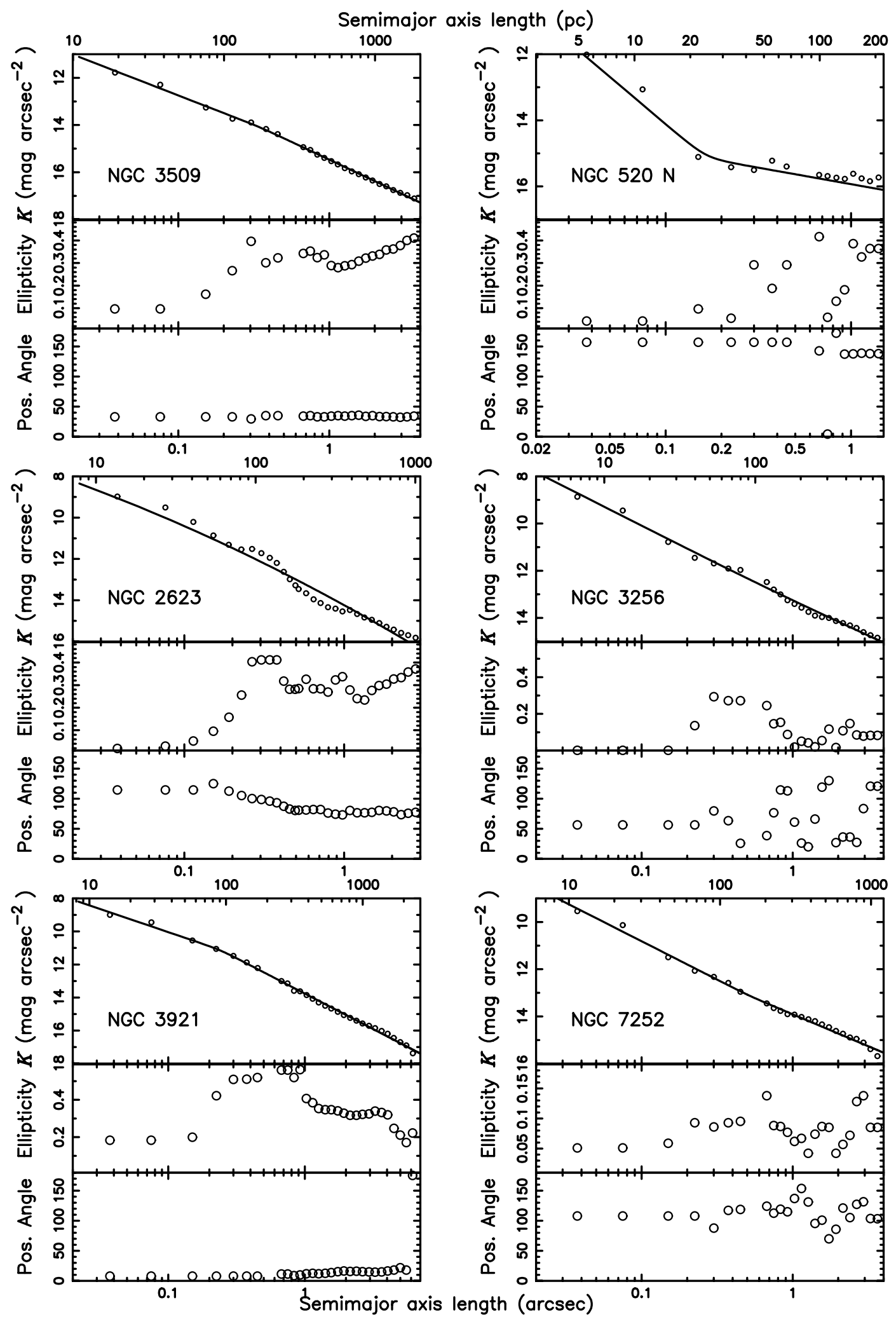

FIG. 23-Continued

clearly visible in the $K$-band image (Fig. 12). This profile and nuclear morphology are very similar to what is seen in the centers of many late-type spiral galaxies (e.g., Böker et al. 2002), where it has been established that the upturn is due to the presence of a nuclear star cluster (e.g., Böker et al. 2004; Rossa et al. 2006). Since more than half of the spiral galaxies have such nuclear star clus- ters, it would not be surprising to find one among the Toomresequence galaxies as well.

Because the galaxies at the earliest stage in the Toomre sequence have not yet experienced the full merger evolution, their nuclei would be expected to still have brightness profiles similar to those of spiral galaxies. At HST resolution, the "classical" $R^{1 / 4}$ 
TABLE 6

Surface Brightness Profile Fitting Parameters

\begin{tabular}{|c|c|c|c|c|c|c|c|c|}
\hline $\begin{array}{l}\text { Galaxy } \\
\text { (1) }\end{array}$ & $\begin{array}{c}\mu_{b} \\
\left(\mathrm{~K} \mathrm{mag} \operatorname{arcsec}^{-2}\right) \\
(2)\end{array}$ & $\begin{array}{c}r_{b} \\
(\operatorname{arcsec}) \\
(3)\end{array}$ & $\begin{array}{l}r_{b} \\
(\mathrm{pc}) \\
(4)\end{array}$ & $\begin{array}{c}\alpha \\
(5)\end{array}$ & $\begin{array}{c}\beta \\
(6)\end{array}$ & $\begin{array}{c}\gamma \\
(7)\end{array}$ & $\begin{array}{c}\Gamma_{0.15} \\
(8)\end{array}$ & $\begin{array}{c}M_{K} \\
(\mathrm{mag}) \\
(9)\end{array}$ \\
\hline 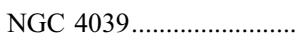 & 13.71 & 0.547 & 36.6 & 0.13 & 1.46 & 0.80 & 1.10 & -23.78 \\
\hline 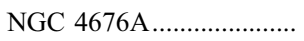 & 14.31 & 0.429 & 183.4 & 0.10 & 1.01 & 0.87 & 0.65 & -24.34 \\
\hline 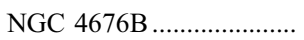 & 14.80 & 1.738 & 743.0 & 4.18 & 1.86 & 0.65 & 0.94 & -24.13 \\
\hline 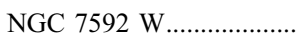 & 14.07 & 0.576 & 271.3 & 10.00 & 1.57 & 1.58 & 1.58 & -24.19 \\
\hline 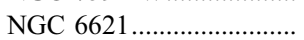 & 13.11 & 0.366 & 146.6 & 10.00 & 0.92 & 0.68 & 0.68 & -24.72 \\
\hline NGC $6622 \ldots \ldots \ldots \ldots \ldots \ldots \ldots \ldots$ & 11.63 & 0.112 & 46.9 & 2.58 & 1.31 & 0.24 & 0.97 & -24.72 \\
\hline 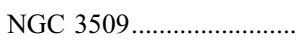 & 14.13 & 0.355 & 176.7 & 10.00 & 1.21 & 0.99 & 0.99 & -24.87 \\
\hline NGC 520 N......................... & 15.04 & 0.165 & 24.4 & 10.00 & 0.40 & 1.90 & 1.49 & -23.73 \\
\hline 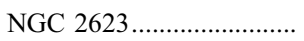 & 13.10 & 0.531 & 190.0 & 0.18 & 3.21 & 0.00 & 1.42 & -24.22 \\
\hline NGC 3256 N........................ & 12.72 & 0.673 & 119.1 & 1.79 & 1.18 & 1.30 & 1.29 & -24.72 \\
\hline 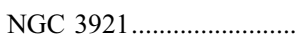 & 11.16 & 0.242 & 91.2 & 10.00 & 1.72 & 1.12 & 1.13 & -25.13 \\
\hline 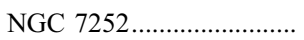 & 13.12 & 0.517 & 156.5 & 10.00 & 1.08 & 1.30 & 1.30 & -24.84 \\
\hline
\end{tabular}

Notes.-Col. (1): Galaxy name. Col. (2): Surface brightness in the $K$ band at the break radius (i.e., the surface brightness $\mu_{b}$ corresponding to the luminosity surface density $\Sigma_{b}$ in eq. [1]). Cols. (3) and (4): Break radius of the surface brightness profile fitting in arcseconds and parsecs, respectively. Cols. (5)-(7): Fit parameters. Column (8): Calculated power-law slope at a radius of $0.15^{\prime \prime}$. Col. (9): Total absolute $K$-band magnitudes, calculated from the apparent $K$-band magnitudes from NED. The majority of the apparent $K$-band magnitudes are from 2MASS. The ones for the last four merger remnants were taken from Rothberg \& Joseph (2006).

bulges in early-type spirals tend to have power-law brightness profiles with slopes $0.5 \leq \gamma \leq 1$ (e.g., Seigar et al. 2002). This is indeed similar to what we find in the earliest stage mergers of the Toomre sequence (NGC 4039, NGC 4676A, and NGC 4676B).

\subsection{Comparison to Elliptical Galaxies}

As merger remnants may evolve into elliptical galaxies, it is of interest to compare the nuclear properties of these two classes of galaxies. We used the results of R01 as a suitable comparison sample of elliptical and $\mathrm{S} 0$ galaxies. While many other HST studies have addressed the surface brightness profiles of early-type galaxies in the optical regime, the R01 study is one of the few that use observations with NICMOS in the NIR. We transformed their $H$-band results to the $K$ band assuming a fixed color $H-K=$ 0.215 , as appropriate for normal early-type galaxies (see Fig. 17).

Previous studies have found that the properties of the nuclear brightness profiles of elliptical galaxies correlate strongly with the total galaxy luminosity (e.g., Faber et al. 1997). To compare the properties of merger remnants and elliptical galaxies, we therefore calculated the total absolute $K$-band magnitudes $\left(M_{K}\right)$ for both our own sample and that of R01, using the apparent $K$-band magnitudes from the Two Micron All Sky Survey (2MASS) and distances based on the observed systemic velocity. The results for our own sample are listed in Table 6.

Figure 24 shows $\Gamma_{0.15}$, the power-law slope at $0.15^{\prime \prime}$, versus $M_{K}$. Optical studies have found that galaxies with $M_{V} \gtrsim-20.5$ $\left(M_{K} \gtrsim-23.6\right)^{13}$ are generally power-law galaxies, that galaxies with $M_{V} \lesssim-22.0\left(M_{K} \lesssim-25.1\right)$ are generally core galaxies, and that galaxies of intermediate luminosities can be of either type (e.g., Faber et al. 1997). The data from R01, shown in Figure 24, support these trends (e.g., beyond $M_{K} \sim-24.7$ there are only core galaxies in their sample, but no power-law galaxies). While these trends appear more pronounced in some studies of other samples (e.g., Faber et al. 1997), it is clear that the correlation has significant scatter.

Figure 24 also shows the Toomre-sequence nuclei in comparison to the E/S0 sample. As discussed already in $\S 5.2$, the Toomre

\footnotetext{
${ }^{13}$ The absolute $K$-band magnitudes listed in parentheses assume $V-K=3.1$, as typical for an old stellar population (Bruzual \& Charlot 2003).
}

nuclei correspond almost exclusively to power-law profiles; only NGC 6622 may possibly be a core-type galaxy. The Toomresequence galaxies occupy the range of absolute magnitudes $M_{K}$ in which both power-law E/S0 and core-type $\mathrm{E} / \mathrm{S} 0$ galaxies can be found; it is therefore no surprise to find power-law profiles among the nuclei of the Toomre-sequence galaxies. However, it is of interest that so few of the nuclei have a core-type profile and that many have $\Gamma_{0.15}$ values that are high relative to those of E/S0 galaxies. We comment on this below in the context of popular theories for the formation of cores in E/S0 galaxies.

In addition, we have studied the luminosity surface density $\Sigma$ at $100 \mathrm{pc}$ as a function of the total absolute $K$-band magnitude,

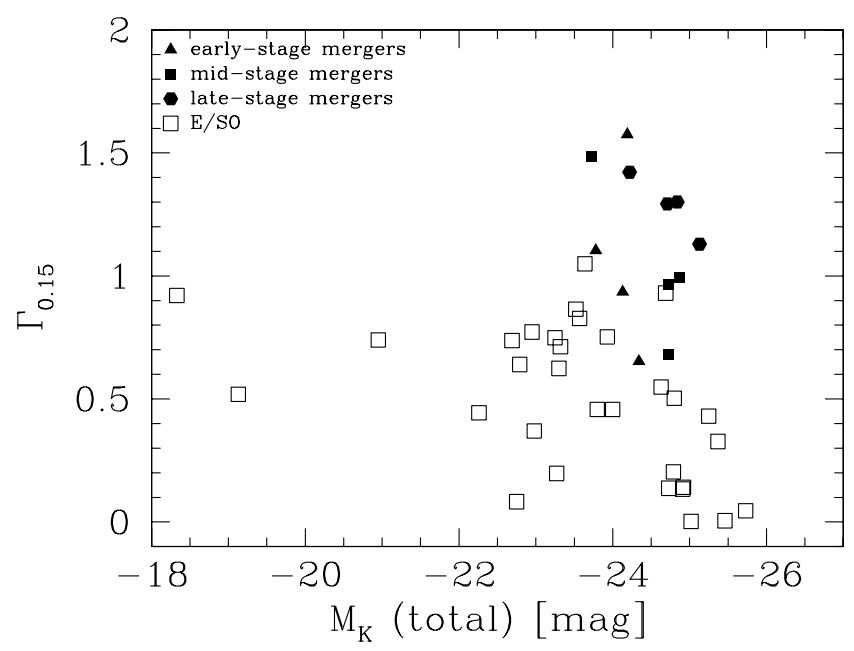

FIG. 24.- Relation between the best-fit power-law slope at $0.15^{\prime \prime}\left(\Gamma_{0.15}\right)$ and the total absolute $K$-band magnitude. For comparison, the early-type E/S0 galaxies from the R01 sample are plotted as open squares. The random uncertainties in $\Gamma_{0.15}$ are small. However, the true uncertainties in $\Gamma_{0.15}$ are dominated by systematic uncertainties that are difficult to quantify, having to do primarily with the accuracy of PSF deconvolution. Nonetheless, these uncertainties can be kept to a minimum by measuring $\Gamma$ at a radius larger than the FWHM of HST's PSF. For the adopted radius of $0.15^{\prime \prime}$ (i.e., $\Gamma_{0.15}$ ), the uncertainties are small enough so as to not affect the overall distribution of points in the plot. The random uncertainties in the absolute magnitudes $M_{K}$ are generally smaller than the symbol sizes. The true uncertainties in $M_{K}$ are dominated by uncertainties in the galaxy distances. 


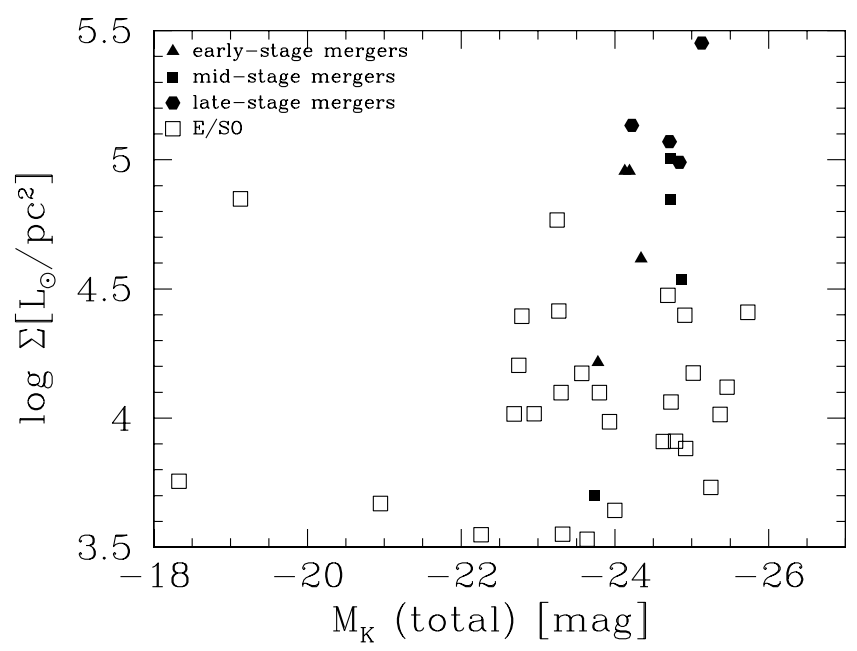

FIG. 25.-Relation between the luminosity surface density $\Sigma$ and the total absolute $K$-band magnitude. The random uncertainties in $\Sigma$ are small. However, the true uncertainties in $\Sigma$ are dominated by systematic uncertainties that are difficult to quantify, having to do primarily with the accuracy of PSF deconvolution. Nonetheless, these uncertainties can be kept to a minimum by measuring $\Sigma$ at a radius larger than the FWHM of HST's PSF. For the adopted radius of $100 \mathrm{pc}$, the uncertainties are small enough so as to not affect the overall distribution of points in the plot. The symbols are as in Figure 24, which also discusses the uncertainties in $M_{K}$.

shown in Figure 25. The majority of the Toomre nuclei have a higher luminosity surface density than the E/S0 galaxies, although there are a few in the overlapping region. The most luminous Toomre galaxies also tend to have the highest surface luminosity density. Note that neither sample was corrected for dust extinction in the $K$ band (see $\S 4.3$ for a discussion of the typical amount of extinction for the Toomre nuclei). However, Figure 17 shows that the Toomre nuclei have more dust extinction than typical E/S0 galaxies. Therefore, any correction for dust extinction would in fact increase the systematic difference in luminosity surface density between the two samples.

Figure 24 shows that, on the whole, the Toomre-sequence nuclei tend to have slopes that are significantly steeper than what is seen in E/S0 galaxies. The four latest-stage merger remnants all have $1.1 \lesssim \Gamma_{0.15} \lesssim 1.4$. In principle this could be a resolution effect, as the median distance of the Toomre galaxies is $77.8 \mathrm{Mpc}$, versus 17.0 Mpc for the galaxies in the sample of R01. Earlytype galaxies in general tend to have steeper logarithmic surface brightness profiles at larger radii, so that at a fixed angular scale, one would naturally expect to find somewhat steeper slopes in a more distant sample. However, Figure 25 shows that this is unlikely to be the whole explanation in the present case. The Toomre nuclei tend to have a higher luminosity surface density than the E/S0 galaxies at fixed physical radius (i.e., at $100 \mathrm{pc}$ ), and thus must be intrinsically brighter at small radii. It then seems plausible that they have intrinsically steeper surface brightness slopes as well. A similar result was recently reported in an HST WFPC2 optical study by Yang et al. (2004), who found that E+A galaxies (shown to be evolved merger remnants) had power-law profiles and higher surface brightnesses than E/S0 galaxies. Yang et al. (2004) attributed the higher surface brightnesses to the recent star formation in $\mathrm{E}+\mathrm{A}$ galaxies and argued that, once the A stellar population faded, the $\mathrm{E}+\mathrm{A}$ galaxies would have surface brightness profiles typical of E/S0 galaxies. Our results also connect naturally with the finding of Rothberg \& Joseph (2004) that many merger remnants have higher luminosity densities near their centers than would be expected on the basis of inward extrapolation of the surface brightness profiles at large radii.
The steep $K$-band profiles and high luminosity surface densities seen in the Toomre galaxies cannot be attributed to the presence of bright nonthermal point sources. We measure the profile slope at $0.15^{\prime \prime}$ and the luminosity surface density at $100 \mathrm{pc}$; the latter corresponds to $0.26^{\prime \prime}$ at the median distance of the sample galaxies. Therefore, both of these quantities are defined outside of the direct influence of a central nonthermal point source, if one exists. This is true in particular because we measure these quantities on the deconvolved profiles, not the observed profiles. Moreover, there is little independent reason to suspect the presence of nonthermal point sources. Most of the surface brightness profiles do not show an upturn near $0.1^{\prime \prime}$. The only exception is NGC $520 \mathrm{~N}$, but for this galaxy a nuclear star cluster is the likely cause. While some of the galaxy images (e.g., NGC 2623 and NGC 3921) do show the NICMOS PSF diffraction pattern around the galaxy center, this merely implies that the galaxy light must be very centrally concentrated (as indeed our analysis confirms), not that a central point source need be present. Finally, nonthermal point sources at optical wavelengths are generally associated with powerful AGNs, and radio galaxies in particular (Verdoes Kleijn et al. 2002). By contrast, most of the Toomre galaxies do not host strong AGNs (see Table 1).

The results of our surface brightness profile analysis have a natural explanation in the context of our understanding of the merger process. As two galaxies interact, gravitational torques remove angular momentum from the gas and drive nuclear inflows (Noguchi 1988; Mihos \& Hernquist 1996; Barnes \& Hernquist 1996). This transports gas to the centers of the interacting galaxies, and subsequently to the center of the merger remnant, triggering nuclear starbursts. The newly formed stars increase the stellar density in the nucleus and steepen the surface mass density profile (Mihos \& Hernquist 1994). Since the young stellar population will have a lower mass-to-light ratio than the older stars in the galaxy, the steepening of the projected luminosity profile will be even stronger. All this is qualitatively consistent with the results shown in Figures 24 and 25. The formation of new stars in a progressing merger may also be responsible for the observed trend of increasing nuclear luminosity with advancing merger stage shown in Figure 21. NGC 3921 and NGC 7252 are known to have "poststarburst" A-star features in spectra of their nuclei, which provides direct evidence that stars recently formed in these mergers (Schweizer 1982; Liu \& Kennicutt 1995a, 1995b).

To quantitatively compare the mass densities of ongoing mergers and elliptical galaxies on the basis of their luminosity densities, one needs to take into account potential differences in the ages of their stellar populations, as well as any radial gradients in the average age within the galaxies. This is not possible in detail here, as we have no estimates available of the stellar population ages in the Toomre-sequence galaxies. However, a simple estimate can be made on the basis of the fact that the dynamical merging age of several of the late-stage Toomre-sequence galaxies has been estimated to be on the order of 1 Gyr (e.g., Hibbard \& van Gorkom 1996). A stellar population fades in the $K$ band by $\sim 1.5$ mag between ages of $10^{9}$ and $10^{10} \mathrm{yr}$ (Bruzual \& Charlot 2003), corresponding to 0.6 dex in luminosity. As the nuclei likely contain a mix of young and old stellar populations, this represents an upper limit to the amount of fading the nuclei may experience. Such significant fading would be sufficient to explain much of the excess luminosity density compared to $\mathrm{E} / \mathrm{S} 0$ galaxies for most of the Toomre-sequence nuclei (see Fig. 25). Also, simulations show that the young stars formed in mergers are likely to be more concentrated toward the center than the underlying older stars. A fading of the young population with time will therefore decrease not only the amplitude but also the power-law slope of the total 
projected luminosity density profile, moving the merger remnants toward the locus of E/S0 galaxies in Figure 24. The effects combined suggest that, if left to evolve for several more gigayears, it is quite possible that the properties of the Toomresequence nuclei would look more similar to the nuclei of normal E/S0 galaxies. The Toomre-sequence remnants would then populate the steep-power-law, high-luminosity-density ends of the E/S0 distributions shown in Figures 24 and 25, respectively.

One possible surprise is that the observed surface brightness profiles are smooth and do not show any obvious kink or upturn. This differs from the profiles obtained in the merger simulations of Mihos \& Hernquist (1994) and later by Springel (2000), which had a pronounced "cusp" inward of a few hundred parsecs due to the presence of the young starburst population (although see Springel et al. [2005] for a discussion of how black hole feedback may moderate these effects). Most of the surface brightness profiles presented here cover radii in the $10 \mathrm{pc}$ to $1 \mathrm{kpc}$ range, so we would have detected any such cusps if they existed. However, it should be noted that pronounced kinks are not necessarily a generic feature of the merger simulations. They can be artifacts of the limited resolution of the simulations, and their possible presence depends sensitively on the adopted (very uncertain) prescriptions for star formation and feedback. In any case, our observations indicate that if there is indeed a centrally concentrated population of young stars, then either its brightness profile connects seamlessly with that of the underlying older stars, or it is more extended (beyond the kiloparsec range) than was predicted in the Mihos \& Hernquist (1994) models.

A predominant theory for the formation of cores in elliptical galaxies is through the evolution of supermassive black holes involved in a merger. If the two merging galaxies both host a central black hole, then these black holes will sink to the center of the merger remnant through dynamical friction and form a binary system that interacts with the surrounding stars. These interactions lead to a hardening of the binary and an ejection of stars from the galaxy center, thus excavating a core (e.g., Milosavljević \& Merritt 2001). The formation of the core happens quickly, in $10^{6}-10^{7} \mathrm{yr}$, so that in this scenario one might expect to find cores in late-stage merger remnants. In fact, there is little evidence for cores in the latest stage mergers of the Toomre sequence. However, our findings do not rule out this scenario, for several reasons: (1) the original merging partners may not both have had black holes; (2) a core may be present but may not be of sufficient size to resolve with our observations; (3) the timescale for black hole binary coalescence may have been shortened substantially by the presence of copious amounts of gas (Escala et al. 2005), so that there was little time for the scattering of stars and the formation of a core; (4) a core may have formed but have been subsequently filled in by stars formed from gas driven to the galaxy center during the merger; or (5) perhaps some minimum galaxy mass is essential for the formation of a core, and the Toomre systems are not massive enough. Note with respect to the last point that galaxy luminosity may not be a good proxy for galaxy mass. The Toomre-sequence galaxies do occupy the same range of absolute magnitudes $M_{K}$ in which E/S0 galaxies are sometimes found to have core profiles. Nonetheless, if the Toomre-sequence galaxies have younger populations on a galaxywide scale (and not just on the nuclear scale discussed above), the fading of these young populations would make the galaxy fainter with time. One should then compare to E/S0 galaxies that are fainter in $M_{K}$, which have core-type profiles much more rarely.

\section{SUMMARY}

We have imaged the central regions of the 11 interacting and merging galaxies of the Toomre sequence in $J, H$, and $K$, using the NIC2 camera on HST NICMOS, and augmented these data with $H S T$ archival data in similar bands for those galaxies with existing NIR data. Compared to optical data, NIR data have the advantage that the images are much less affected by dust. The observations therefore offer a relatively unimpeded view into the nuclei, especially when compared to the results of our previous HST WFPC2 study presented in Paper I. With our previous optical data it was difficult or impossible in several cases to identify the true galaxy nucleus/nuclei; by contrast, the NIR data presented here generally reveal the nuclei clearly. In most cases we confirm the galaxy nucleus/nuclei reported from data in other wave bands, such as radio, X-ray, or millimeter (CO) wavelengths. The nuclear positions identified from the different observations are generally consistent with each other. In NGC 7764A we detect a double nucleus for the first time, with a separation of $0.43^{\prime \prime}$ ( $\left.260 \mathrm{pc}\right)$, similar to the separation of the two nuclei in the merging galaxy Arp 220. The presence of a double nucleus in NGC 7764A is consistent with Toomre's initial assumption of the system being in the final merging stage prior to coalescence. In NGC $3256 \mathrm{~S}$ we find a highly extincted NIR source coincident with the secondary nucleus previously identified on the basis of radio and X-ray data. The extinction-corrected $K$-band luminosity of this source is similar to that of the nuclei in other Toomre-sequence galaxies.

Aperture photometry of the Toomre-sequence nuclei reveals that their colors are consistent with the observed colors of elliptical galaxies and the predicted colors of intermediate-age or old stellar populations, but often with considerable extinction. There is a marginal trend for the nuclei to become bluer as a function of the merger stage in the Toomre sequence. Because stellar population differences alone cannot account for the range of colors we observe in the nuclei, we attribute this trend to a dispersal of dust during the late stages of merging. This is qualitatively consistent with the predictions of models of merging galaxies with black holes in which AGN feedback is invoked to disperse obscuring dust as the merger progresses (e.g., Hopkins et al. 2005). It will be valuable to construct numerical models that make quantitative predictions for the dust and gas content of interacting and merging galaxies as a function of merger (st)age, for comparison to our data.

There is also a trend for the Toomre-sequence nuclei to become more luminous (within an aperture of fixed physical size, and after correction for extinction) as a function of the merger stage along the sequence. As long as there are no systematic errors in the analysis which depend on the merger stage, this trend is statistically significant at the $2 \sigma$ level. This result suggests that, as the merger proceeds, either the stellar density must increase or the average stellar age must decrease (or both). As a cautionary note we remark that this result is based on the assumed merger stage, which may not necessarily reflect the true merger (st)age, given how the Toomre sequence was initially defined in the first place. In principle it is possible to test these scenarios in more detail using population synthesis models to fit the broadband colors. However, the results of such an analysis are likely to be quite uncertain given the large amounts of extinction and the uncertainties in the dust geometry. We therefore postpone a discussion of the stellar populations to a subsequent paper that will present HST STIS spectra, obtained in the context of our investigation.

We have calculated radial $V-K$ color gradients around all the nuclei in our sample. The latest two merger remnants have rather shallow color gradients, whereas most other nuclei have steeper gradients. The nuclei with the strongest color gradients are mostly found in the midstage mergers. Several of the nuclei become considerably redder inward of about $5^{\prime \prime}$ from the nucleus, indicating strong concentrations of dust in the nuclei. 
Finally, we have derived the $K$-band surface brightness profiles for those Toomre-sequence nuclei for which the morphology allows a meaningful isophotal analysis (12 out of the total 18). The profiles were fit with a so-called Nuker law to facilitate a comparison with other samples of galaxies observed with HST. The majority of the nuclei have steep profiles that can be characterized as power-law profiles. In general, the Toomre-sequence galaxies tend to have steeper profiles and higher central luminosity surface densities than E/S0 galaxies. These findings can both be qualitatively explained if the Toomre-sequence galaxies have young stellar populations concentrated toward their centers. Such populations are expected on the basis of $N$-body simulations of spiral galaxy mergers, in which gas flows toward the center of the merger remnant trigger nuclear starbursts. This process may also be responsible for the observed trend in the Toomre sequence of increasing nuclear luminosity with advancing merger stage. If left to evolve and fade for several more gigayears, it is quite possible that the properties of the Toomre-sequence nuclei would look similar to the nuclei of normal $\mathrm{E} / \mathrm{S} 0$ galaxies. Our results therefore support the view that mergers of spiral galaxies lead to the formation of early-type galaxies.

Support for proposal 9402 was provided by NASA through a grant from the Space Telescope Science Institute, which is op- erated by the Association of Universities for Research in Astronomy, Inc., under NASA contract NAS5-26555. We thank Nick Scoville for kindly providing us with the drizzled NGC 2623 images, and Daisuke Iono for providing us with the unpublished coordinates of the CO measurements of the nuclei of NGC 6621 and NGC 7592. We would also like to thank the anonymous referee for a very constructive and detailed report, which helped to improve the clarity and contents of the paper. R. P. v. d. M. carried out part of this research at the Kavli Institute for Theoretical Physics in Santa Barbara, supported in part by the National Science Foundation under grant PHY 99-07949. A. I. Z. is grateful for the hospitality and support of the Aspen Center for Physics, the Kavli Institute for Theoretical Physics, and the New York University Physics Department and Center for Cosmology and Particle Physics during her sabbatical year. This research has made use of the NASA/IPAC Extragalactic Database, which is operated by the Jet Propulsion Laboratory, California Institute of Technology, under contract with the National Aeronautics and Space Administration. This publication makes use of data products from the Two Micron All Sky Survey, which is a joint project of the University of Massachusetts and the Infrared Processing and Analysis Center/California Institute of Technology, funded by the National Aeronautics and Space Administration and the National Science Foundation.
Aldcroft, T. 2002, Chandra Absolute Astrometric Accuracy (Cambridge: CXC), http://cxc.harvard.edu/cal/ASPECT/celmon/

Arp, H. C. 1966, Atlas of Peculiar Galaxies (Pasadena: Caltech)

Barnes, J. E. 1988, ApJ, 331, 699

Barnes, J. E., \& Hernquist, L. 1996, ApJ, 471, 115

Bessell, M. S., \& Brett, J. M. 1988, PASP, 100, 1134

Beswick, R. J., Pedlar, A., Clemens, M. S., \& Alexander, P. 2003, MNRAS, 346, 424

Binney, J. J., \& Merrifield, M. 1998, Galactic Astronomy (Princeton: Princeton Univ. Press)

Böker, T., Laine, S., van der Marel, R. P., Sarzi, M., Rix, H.-W., Ho, L. C., \& Shields, J. C. 2002, AJ, 123, 1389

Böker, T., Sarzi, M., McLaughlin, D. E., van der Marel, R. P., Rix, H.-W., Ho, L. C., \& Shields, J. C. 2004, AJ, 127, 105

Böker, T., Storey, J. W. V., Krabbe, A., \& Lehmann, T. 1997, PASP, 109, 827

Borne, K. D., Colina, L., Bushouse, H., \& Lucas, R. A. 1999, ApJ, 527, 554

Borne, K. D., \& Richstone, D. G. 1991, ApJ, 369, 111

Braine, J., Duc, P.-A., Lisenfeld, U., Charmandaris, V., Vallejo, O., Leon, S., \& Brinks, E. 2001, A\&A, 378, 51

Bruzual, G., \& Charlot, S. 2003, MNRAS, 344, 1000

Bryant, P. M., \& Scoville, N. Z. 1999, AJ, 117, 2632

Bushouse, H. A., \& Stanford, S. A. 1992, ApJS, 79, 213

Bushouse, H. A., \& Werner, M. W. 1990, ApJ, 359, 72

Byun, Y.-I., et al. 1996, AJ, 111, 1889

Carollo, C. M., Stiavelli, M., de Zeeuw, P. T., \& Mack, J. 1997, AJ, 114, 2366

Carral, P., Turner, J. L., \& Ho, P. T. P. 1990, ApJ, 362, 434

Casoli, F., Dupraz, C., Combes, F., \& Kazes, I. 1991, A\&A, 251, 1

Chabrier, G. 2003, PASP, 115, 763

Condon, J. J., Helou, G., Sanders, D. B., \& Soifer, B. T. 1990, ApJS, 73, 359 1996, ApJS, 103, 81

Dickinson, M. E., et al. 2002, in HST NICMOS Data Handbook, ver. 5.0, ed. B. Mobasher (Baltimore: STScI), 3-1

Escala, A., Larson, R. B., Coppi, P. S., \& Mardones, D. 2005, ApJ, 630, 152

Fabbiano, G., Kim, D.-W., \& Trinchieri, G. 1992, ApJS, 80, 531

Fabbiano, G., Schweizer, F., \& Mackie, G. 1997, ApJ, 478, 542

Fabbiano, G., Zezas, A., \& Murray, S. S. 2001, ApJ, 554, 1035

Faber, S. M., et al. 1997, AJ, 114, 1771

Ferrarese, L., et al. 2006, ApJS, 164, 334

Georgakakis, A., Forbes, D. A., \& Norris, R. P. 2000, MNRAS, 318, 124

Gerssen, J., van der Marel, R. P., Axon, D., Mihos, J. C., Hernquist, L., \& Barnes, J. E. 2004, AJ, 127, 75

Hibbard, J. E., Guhathakurta, P., van Gorkom, J. H., \& Schweizer, F. 1994, AJ, 107,67

Hibbard, J. E., van der Hulst, J. M., Barnes, J. E., \& Rich, R. M. 2001, AJ, 122, 2969

Hibbard, J. E., \& van Gorkom, J. H. 1996, AJ, 111, 655
Hibbard, J. E., \& Yun, M. S. 1999, ApJ, 522, L93

Hopkins, P. F., Hernquist, L., Cox, T. J., Di Matteo, T., Robertson, B., \& Springel, V. 2006, ApJS, 163, 1

Hopkins, P. F., Hernquist, L., Martini, P., Cox, T. J., Robertson, B., Di Matteo, T., \& Springel, V. 2005, ApJ, 625, L71

Iono, D., Yun, M. S., \& Ho, P. T. P. 2005, ApJS, 158, 1

Joseph, R. D., Meikle, W. P. S., Robertson, N. A., \& Wright, G. S. 1984, MNRAS, 209, 111

Joseph, R. D., \& Wright, G. S. 1985, MNRAS, 214, 87

Kassin, S. A., Frogel, J. A., Pogge, R. W., Tiede, G. P., \& Sellgren, K. 2003, AJ, 126, 1276

Kennicutt, R. C., Jr., Roettiger, K. A., Keel, W. C., van der Hulst, J. M., \& Hummel, E. 1987, AJ, 93, 1011

Komossa, S., Burwitz, V., Hasinger, G., Predehl, P., Kaastra, J. S., \& Ikebe, Y. 2003, ApJ, 582, L15

Kotilainen, J. K., Moorwood, A. F. M., Ward, M. J., \& Forbes, D. A. 1996, A\&A, 305, 107

Kotilainen, J. K., Reunanen, J., Laine, S., \& Ryder, S. D. 2001, A\&A, 366, 439

Krist, J., \& Hook, R. 2001, The TinyTim User's Guide (Baltimore: STScI)

Laine, S., van der Marel, R. P., Lauer, T. R., Postman, M., O'Dea, C. P., \& Owen, F. N. 2003a, AJ, 125, 478

Laine, S., van der Marel, R. P., Rossa, J., Hibbard, J. E., Mihos, J. C., Böker, T., \& Zabludoff, A. I. 2003b, AJ, 126, 2717 (Paper I)

Lauer, T. R. 1985, ApJS, 57, 473 1986, ApJ, 311, 34

Lauer, T. R., et al. 1995, AJ, 110, 2622 2005, AJ, 129, 2138

Lira, P., Ward, M., Zezas, A., Alonso-Herrero, A., \& Ueno, S. 2002, MNRAS, 330,259

Liu, C. T., \& Kennicutt, R. C., Jr. 1995a, ApJ, 450, 547 1995b, ApJS, 100, 325

Lonsdale, C. J., Persson, S. E., \& Matthews, K. 1984, ApJ, 287, 95

Lucy, L. B. 1974, AJ, 79, 745

McMahon, R. G., White, R. L., Helfand, D. J., \& Becker, R. H. 2002, ApJS, 143,1

Mihos, J. C., \& Hernquist, L. 1994, ApJ, 431, L9

. 1996, ApJ, 464, 641

Milosavljević, M., \& Merritt, D. 2001, ApJ, 563, 34

Neff, S. G., \& Ulvestad, J. S. 2000, AJ, 120, 670

Neff, S. G., Ulvestad, J. S., \& Campion, S. D. 2003, ApJ, 599, 1043

Noguchi, M. 1988, A\&A, 203, 259

Nolan, L. A., Ponman, T. J., Read, A. M., \& Schweizer, F. 2004, MNRAS, 353, 221

Norris, R. P., \& Forbes, D. A. 1995, ApJ, 446, 594

Pasquali, A., Gallagher, J. S., \& de Grijs, R. 2004, A\&A, 415, 103

Ptak, A. F., Colbert, E. J. M., van der Marel, R. P., Roye, E. W., Heckman, T. M., \& Towne, B. 2006, ApJS, 166, 154 
Rafanelli, P., \& Marziani, P. 1992, AJ, 103, 743

Ravindranath, S., Ho, L. C., Peng, C. Y., Filippenko, A. V., \& Sargent, W. L. W. 2001, AJ, 122, 653 (R01)

Read, A. M. 2003, MNRAS, 342, 715

Rest, A., van den Bosch, F. C., Jaffe, W., Tran, H., Tsvetanov, Z., Ford, H. C., Davies, J., \& Schafer, J. 2001, AJ, 121, 2431

Richardson, W. H. 1972, J. Opt. Soc. Am., 62, 55

Rieke, G. H., \& Lebofsky, M. J. 1985, ApJ, 288, 618

Rossa, J., van der Marel, R. P., Böker, T., Gerssen, J., Ho, L. C., Rix, H.-W., Shields, J. C., \& Walcher, C. J. 2006, AJ, 132, 1074

Rothberg, B., \& Joseph, R. D. 2004, AJ, 128, 2098 2006, AJ, 131, 185

Sakamoto, K., Ho, P. T. P., \& Peck, A. B. 2006, ApJ, 644, 862

Sanders, D. B., \& Mirabel, I. F. 1996, ARA\&A, 34, 749

Sanders, D. B., Soifer, B. T., Elias, J. H., Madore, B. F., Matthews, K., Neugebauer, G., \& Scoville, N. Z. 1988, ApJ, 325, 74

Saviane, I., Hibbard, J. E., \& Rich, R. M. 2004, AJ, 127, 660

Schade, D., Micol, A., Durand, D., Pirenne, B., Simard, L., Dolensky, M., \& Stetson, P. B. 2002, The WFPC2 Association Science Products Pipeline at CADC and ST-ECF (Victoria: Canadian Astron. Data Centre), http://archive .eso.org/archive/hst/wfpc2asn/3sites/WFPC2Newsletter.pdf

Schreier, E. J., et al. 1998, ApJ, 499, L143

Schweizer, F. 1982, ApJ, 252, 455 . 1996, AJ, 111, 109

1998, in Galaxies: Interactions and Induced Star Formation, ed. D. Friedli, L. Martinet, \& D. Pfenniger (Heidelberg: Springer), 105

Schweizer, F., Miller, B. W., Whitmore, B. C., \& Fall, S. M. 1996, AJ, 112, 1839

Schweizer, F., Seitzer, P., \& Brodie, J. P. 2004, AJ, 128, 202
Scoville, N. Z., et al. 1998, ApJ, 492, L107 2000, AJ, 119, 991

Seigar, M., Carollo, C. M., Stiavelli, M., de Zeeuw, P. T., \& Dejonghe, H. 2002, AJ, 123,184

Silva, D. R., \& Bothun, G. D. 1998, AJ, 116, 85

Springel, V. 2000, MNRAS, 312, 859

Springel, V., Di Matteo, T., \& Hernquist, L. 2005, MNRAS, 361, 776

Stanford, S. A., \& Bushouse, H. A. 1991, ApJ, 371, 92

Toomre, A. 1977, in The Evolution of Galaxies and Stellar Populations, ed. B. M. Tinsley \& R. B. Larson (New Haven: Yale Univ. Press), 401

Toomre, A., \& Toomre, J. 1972, ApJ, 178, 623

Verdoes Kleijn, G. A., Baum, S. A., de Zeeuw, P. T., \& O’Dea, C. P. 2002, AJ, 123,1334

Wang, Z., Schweizer, F., \& Scoville, N. Z. 1992, ApJ, 396, 510

Weilbacher, P. M., Duc, P.-A., \& Fritze-V. Alvensleben, U. 2003, A\&A, 397, 545

Whitmore, B. C., \& Schweizer, F. 1995, AJ, 109, 960

Whitmore, B. C., Schweizer, F., Leitherer, C., Borne, K., \& Robert, C. 1993, AJ, 106, 1354

Whitmore, B. C., \& Zhang, Q. 2002, AJ, 124, 1418

Whitmore, B. C., Zhang, Q., Leitherer, C., Fall, S. M., Schweizer, F., \& Miller, B. W. 1999, AJ, 118, 1551

Whitmore, B. C., et al. 2005, AJ, 130, 2104

Wright, G. S., James, P. A., Joseph, R. D., \& McLean, I. S. 1990, Nature, 344, 417

Yang, Y., Zabludoff, A. I., Zaritsky, D., Lauer, T. R., \& Mihos, J. C. 2004, ApJ, 607,258

Yun, M. S., \& Hibbard, J. H. 2001, ApJ, 550, 104

Zezas, A., Fabbiano, G., Rots, A. H., \& Murray, S. S. 2002a, ApJ, 577, 710 2002b, ApJS, 142, 239 